\title{
Assessment of Genetic Diversity for Stem Rust and Stripe Rust Resistance in an International Wheat Nursery Using Phenotypic and Molecular Technologies
}

\author{
B. Chemayek ${ }^{1, *}$, U.K. Bansal ${ }^{1}$, H. Miah ${ }^{1}$, W.W. Wagoire ${ }^{2}$, H.S. Bariana ${ }^{1}$ \\ ${ }^{1}$ The University of Sydney Plant Breeding Institute, School of Life and Environmental Sciences. \\ ${ }^{2}$ Buginyanya Zonal Agricultural Research and Development Institute, National Agricultural \\ Research Organisation. \\ *Corresponding author. @ bchemayek@gmail.com
}

\begin{abstract}
The objective of this study was to assess diversity for stem rust and stripe rust resistance in an international wheat screening nursery under greenhouse conditions using pathotypes with known avirulence/ virulence profiles. A set of 95 entries of an international wheat screening nursery collected from material generated by staff of the International Maize and Wheat Improvement Centre (CIMMYT) was tested against seven Australian Pgt and five Pst pathotypes through artificial inoculation under the greenhouse conditions using standard procedures. Ten all-stage stem rust resistance genes ( $\mathrm{Sr} 8 a, \mathrm{Sr} 8 b, \mathrm{Sr} 9 b, \mathrm{Sr} 12, \mathrm{Sr} 17, \mathrm{Sr} 23, \mathrm{Sr} 24, \mathrm{Sr} 30, \mathrm{Sr} 31$ and $\mathrm{Sr} 38$ ) and seven all-stage stripe rust resistance genes ( $\mathrm{Yr} 3, \mathrm{Yr} 4, \mathrm{Yr} 6, \mathrm{Yr} 9, \mathrm{Yr} 17, \mathrm{Yr} 27$ and $\mathrm{Yr} 34)$ were postulated either singly or in combinations based on seedling responses of test entries against pathotypes differing in virulence for commonly deployed genes. Sr30 and $\mathrm{Sr} 38$ were the most common stem rust resistance genes in this nursery. The $\mathrm{Sr} 38$-linked stripe rust resistance gene $\mathrm{Yr} 17$ was present in high proportion. The presence of rust resistance genes $\mathrm{Sr} 24, \mathrm{Sr} 31 / \mathrm{Yr} 9, \mathrm{Sr} 38 / \mathrm{Yr} 17$ and $\mathrm{Yr} 4$ were confirmed using the closely linked molecular markers. The adult plant resistance (APR) genes $\mathrm{Sr} 2$ and $\mathrm{Lr} 34 / \mathrm{Yr} 18 / \mathrm{Sr} 57$ were detected using linked molecular markers $c s S r 2$ and $c s L V 34$, respectively. Genotypes carrying combinations of stem rust and stripe rust resistance were identified for use as donor sources in breeding programs.
\end{abstract}

Keywords: Gene, Host resistance, Stem rust, Stripe rust, Wheat.

\section{Introduction}

Rust diseases of wheat are among the most important production constraints in all wheat growing regions globally (McIntosh et al., 1995; Roelfs et al., 1992). Stem rust caused by Puccinia graminis f. sp. tritici (Pgt) and stripe rust caused by P. striiformis f. sp. tritici (Pst) can cause up to 100\% yield loss on susceptible cultivars (Roelfs et al., 1992; McIntosh et al., 1995; Chen, 2005; Bansal et al., 2014). Frequent emergence and rapid spread of more virulent and aggressive pathotypes of Pgt (Pretorius et al., 2000; Nazari et al., 2009) and Pst (Ali et al., 2017, Chen, 2020; Chen et al., 2014; Wellings, 2011; FAO, 2014) continue to pose a serious threat to global food 
security (Singh et al., 2011a, Chen, 2020). The emergence of highly virulent pathotypes of Pgt in the East African highlands combines unique and complex virulence defeating many resistance genes previously effective against local Pgt pathotypes in individual geographies (Vikram et al., 2021). The detection of highly aggressive Pgt pathotypes in Uganda in 1999 (Ug99) rendered more than $80 \%$ of global wheat varieties susceptible (Singh et al., 2011a). Over the past decade 14 variants within the $\mathrm{Ug} 99$ race group have emerged and spread across East African countries (Singh et al., 2011a, Bhavani et al., 2019). The rapid evolution, spread and aggressive nature of new Pst pathotypes in major wheat growing countries around the world has made it the most significant disease (Ali et al., 2017, Vikram et al., 2021). Stripe rust has historically been endemic to areas with humid and cool summers or in warm high-altitude areas with cool nights but in recent years, stripe rust has shown greater adaptation in warmer areas, where the disease was previously less important (Ali et al., 2017, Vikram et al., 2021). Repeated incursions of new pathotypes at national or continental scales have been reported (Ali et al., 2014, Markell et al., 2008, Walter et al., 2016). High rates of mutation from avirulence to virulence (Hovmøller and Justesen, 2007) have contributed to increased susceptibility of varieties over area and time (Milus et al., 2015, Sørensen et al., 2014, Vikram et al., 2021).

Various control options are available to minimize losses caused by rust pathogens. Fungicides effectively control stem rust (Wanyera et al., 2009; Tadesse et al., 2010; Macharia et al., 2013) and stripe rust (Carmona et al., 2020; Wellings, 2011; Murray and Brennan, 2009). The global cost of controlling wheat stripe rust using fungicides is at least \$US 1 billion annually (Chen, 2020). The use of fungicides in Australia reduced losses from stripe rust by A $\$ 359$ million, annually (Murray and Brennan, 2009). In China, about 6 million hectares of wheat are treated with fungicides (Kang et al., 2010; Carmona et al., 2020), while in the US Pacific Northwest, wheat growers spend at least \$US 10 million on use of fungicides to control stripe rust every year (Chen, 2020). This is an expensive method of rust control; especially for small scale farmers worldwide (Oliver, 2014). Host plant resistance is the most effective, economical and eco-friendly method of controlling wheat rust diseases (Bariana et al., 2007a; Qamar et al., 2008; Vanzetti et al., 2011; Bansal et al., 2015). Long term success in breeding for triple rust resistance is influenced by knowledge of pathotypic evolution, availability of genetically diverse sources of natural resistance, and the access to high throughput screening methodologies (Bariana et al., 2007a; Singh et al., 2011a).

Knowledge of genetic basis of host resistance in wheat cultivars, high throughput screening and a well-developed pre-breeding pipelines form the basis for successful breeding (Bariana, 2003; Bariana et al., 2007a; Admassu et al., 2012; Bansal et al., 2015). Host resistance is categorized into two types; seedling resistance also called all stage resistance (ASR) and adult plant resistance (APR) (Bariana, 2003; Chen, 2005; Bariana et al., 2007a; Kou and Wang, 2010; Ellis et al., 2014). ASR is controlled by genes with major effects, and it is often short lived as it is prone to be matched by evolution of virulence in pathogen populations. Durability of this resistance can be achieved by pyramiding more than two genes in new cultivars (Bariana et al., 2007a; Bernardo et al., 2012; Ellis et al., 2014). On the other hand, APR is controlled by genes with small effects that express at the post seedling stages (Bariana, 2003). A combination of more than two APR genes is essential to achieve acceptable levels of resistance (Bariana and McIntosh, 1995; Singh et al. 2011b). Deployment of combinations of 4-5 APR genes confers 'near-immune' resistance and lasts for a longer time (Singh et al., 2011b; Singh et al., 2014). Hence, achievement of durable control of wheat rust diseases requires identification, characterization and deployment of combinations of diverse sources of resistance (Kolmer et al., 2007; Bariana et al., 2007a, 2007b; Admassu et al., 2012). Advances in molecular marker technology and availability of gene-linked or gene-specific markers ensure efficient pyramiding 
of rust resistance genes (Kolmer et al., 2013). Molecular markers have been developed for several rust resistance genes (http://maswheat.ucdavis.edu/Index.htm). These markers can be used for the detection of target genes in germplasm collections in the absence of appropriate pathogen isolates.

Tests with an array of pathotypes differing in virulence genes offer the most efficient way to determine the genetic diversity for resistance to a target plant pathogen among a set of germplasm (Singh et al., 2014). Interpretation of results from multi-pathotype testing is based on the gene-for-gene concept in the case of rust diseases. Resistance genes are postulated by comparing infection types (ITs) produced by an array of pathotypes on genotypes under consideration with ITs produced by genotypes carrying known resistance gene(s) (Pathan and Park, 2007; Singh et al., 2008a, Singh et al., 2014). This methodology has been widely used to postulate all stage rust resistance genes in wheat. It requires well characterized pathotypes with diverse combinations of virulence and avirulence profiles and such resources are available in several laboratories (Kolmer, 2003; Pathan and Park, 2007; Admassu et al., 2012; Singh et al., 2014). Field testing of seedling susceptible genotypes enables identification of genotypes carrying APR.

Wheat cultivars derived from the CIMMYT germplasm are grown globally through continuous exchange of material with national research programs (Singh and Rajaram, 2002; Ortiz et al., 2008; Pretorius et al., 2015). CIMMYT breeders incorporate diverse rust resistance genes into elite germplasm. The rust resistant lines with good agronomic traits are compiled into screening nurseries and distributed annually for rust screening in many wheat growing countries (Ortiz et al., 2008). Although wheat lines distributed globally by CIMMYT are selected based on their resistance to the three rust diseases (Singh et al., 2008b), screening of germplasm against the local rust flora is essential. This study was planned to test an international wheat screening nursery against several Australian Pgt and Pst pathotypes in the greenhouse to understand genetic diversity for stem rust and stripe rust resistance.

\section{Materials and Methods}

\section{Host materials}

A set of 95 lines from a CIMMYT (C21SAWYT-AUS) wheat screening nursery was tested in the greenhouse to postulate stem rust and stripe rust resistance genes. Pedigree details are listed in Table 1. Stem rust and stripe rust differential sets with known resistance genes were sown with each experiment. Details of the differentials used are listed in McIntosh et al., (1995).

\section{Pathogen materials}

Wheat genotypes were tested with seven Australian Pgt pathotypes: 34-1,2,3,4,5,6,7 (Culture number 103); 34-1,2,3,6,7,(8),9 (205); 34-1,2,3,5,7,8,9 (206); 343-1,2,3,5,6,(8),9 (465); 98 1,2,(3),(5),6 (279); 34-1,2,7+Sr38 (565); 34-2,4,5,7,11 (99) and five Pst pathotypes: 134 $\mathrm{E} 16 \mathrm{~A}+$ Yr17+ (599); $134 \mathrm{E} 16 \mathrm{~A}+$ Yr17+Yr27 (617); $110 \mathrm{E} 143 \mathrm{~A}+$ (444); $108 \mathrm{E} 141 \mathrm{~A}+$ (420), 104 $\mathrm{E} 137 \mathrm{~A}+(414)$. The avirulence/virulence formulae of different pathotypes used are presented in Table 2. 
Table 1. Pedigree information, postulated genes and molecular marker data for the 95 entries of an international wheat nursery

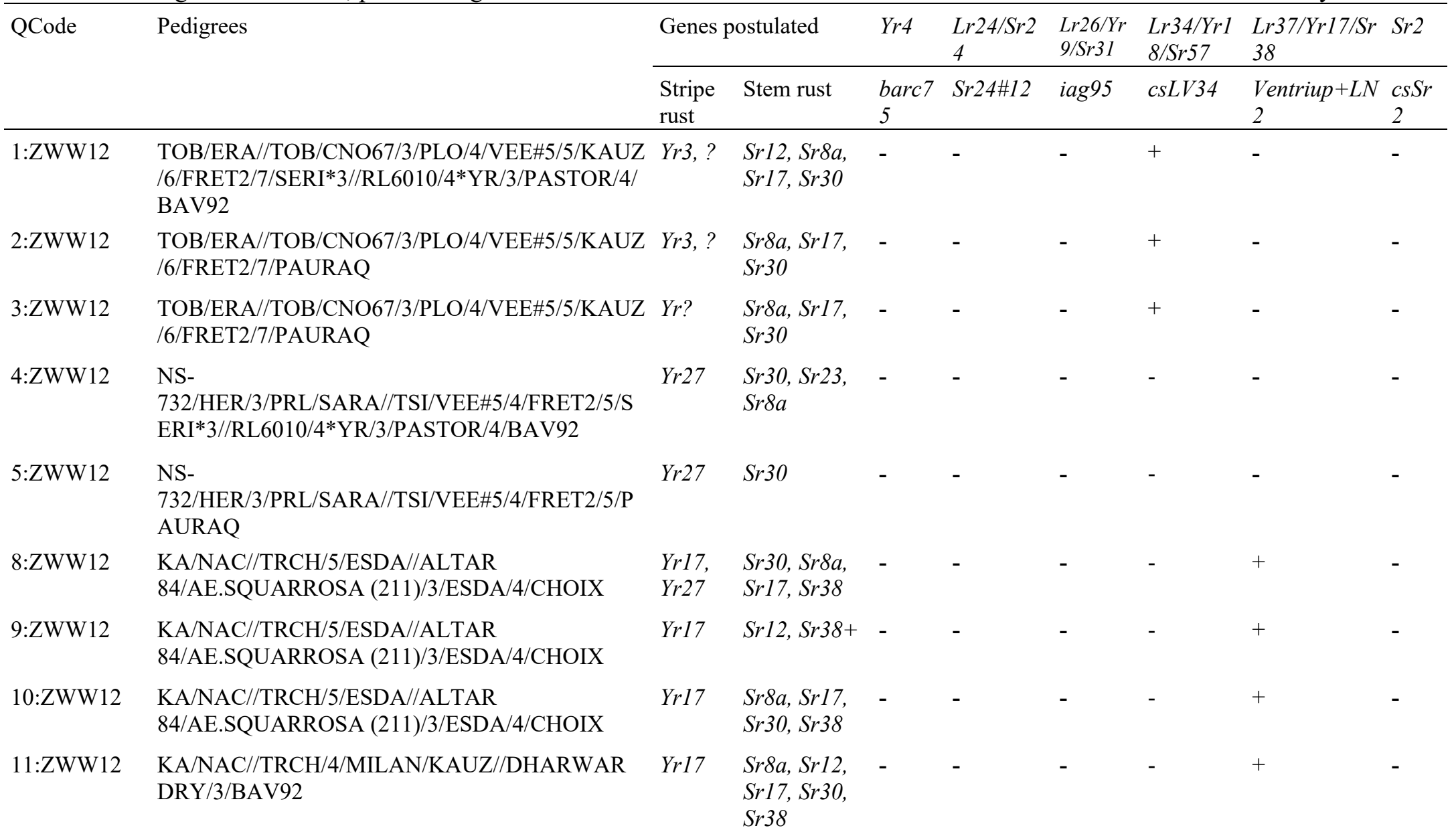




\begin{tabular}{|c|c|c|c|c|c|c|c|c|c|}
\hline \multirow[t]{2}{*}{ QCode } & \multirow[t]{2}{*}{ Pedigrees } & \multicolumn{2}{|c|}{ Genes postulated } & \multirow{2}{*}{$\begin{array}{l}Y r 4 \\
\operatorname{barc7} \\
5\end{array}$} & \multirow{2}{*}{$\begin{array}{l}\operatorname{Lr} 24 / S r 2 \\
4 \\
\operatorname{Sr} 24 \# 12\end{array}$} & \multirow{2}{*}{$\begin{array}{l}\text { Lr26/Yr } \\
9 / \mathrm{Sr} 31 \\
\text { iag95 }\end{array}$} & \multirow{2}{*}{$\begin{array}{l}\operatorname{Lr} 34 / \mathrm{Yr} 1 \\
8 / \operatorname{Sr} 57 \\
\operatorname{csLV} 34\end{array}$} & \multirow{2}{*}{$\begin{array}{l}\text { Lr37/Yr17/Sr } \\
38 \\
\text { Ventriup }+L N \\
2\end{array}$} & \multirow{2}{*}{$\begin{array}{l}S r 2 \\
\operatorname{csS} S \\
2\end{array}$} \\
\hline & & $\begin{array}{l}\text { Stripe } \\
\text { rust }\end{array}$ & Stem rust & & & & & & \\
\hline 12:ZWW12 & $\mathrm{KA} / \mathrm{NAC} / / \mathrm{TRCH} / 3 / \mathrm{MUU}$ & NIL & $\operatorname{Sr} 17, \operatorname{Sr} 30$ & - & - & - & - & - & - \\
\hline 13:ZWW12 & $\mathrm{KA} / \mathrm{NAC} / / \mathrm{TRCH} / 3 / \mathrm{PAURAQ}$ & $Y r ?$ & $\begin{array}{l}\text { Sr8a, Sr12, } \\
\text { Sr17, Sr } 30+\end{array}$ & - & - & - & - & - & - \\
\hline 15:ZWW12 & $\begin{array}{l}\mathrm{KA} / \mathrm{NAC} / / \mathrm{TRCH} / 5 / \mathrm{SERI} * 3 / / \mathrm{RL} 6010 / 4 * \mathrm{YR} / 3 / \mathrm{PAS} \\
\mathrm{TOR} / 4 / \mathrm{BAV} 92\end{array}$ & NIL & $\operatorname{Sr} 30$ & - & - & - & - & - & - \\
\hline 16:ZWW12 & KA/NAC//TRCH/3/PAURAQ & $\operatorname{Yr} 27$ & $\operatorname{Sr} 30$ & - & - & - & - & - & - \\
\hline 17:ZWW12 & KA/NAC//TRCH/3/PAURAQ & $\operatorname{Yr} 3$ & $\operatorname{Sr} 30$ & - & - & - & - & - & - \\
\hline 18:ZWW12 & $\begin{array}{l}\text { FALCIN/AE.SQUARROSA } \\
\text { (312)/3/THB/CEP7780//SHA4/LIRA/4/FRET2/5/A } \\
\text { TTILA*2/PBW65 }\end{array}$ & $Y r ?$ & $\operatorname{Sr} 17+$ & - & - & - & 1 & - & Null \\
\hline 19:ZWW12 & 1447/PASTOR//KRICHAUFF/3/PAURAQ & $\begin{array}{l}\text { Yr17, } \\
\operatorname{Yr} 27\end{array}$ & $\operatorname{Sr} 24, \operatorname{Sr} 38$ & - & + & - & - & + & Null \\
\hline 20:ZWW12 & ANNUELLO/3/KA/NAC//TRCH & $\operatorname{Yr} 3, ?$ & $\operatorname{Sr} 8 a$ & - & - & - & - & - & - \\
\hline 21:ZWW12 & VEE/LIRA//BOW/3/BCN/4/KAUZ/5/DANPHE \#1 & $Y r 3, ?$ & $\operatorname{Sr} 8 b, \operatorname{Sr} 17$ & - & - & - & - & - & - \\
\hline 23:ZWW12 & WBLL1/PAURAQ & NIL & Sr30 & - & - & - & - & - & - \\
\hline 24:ZWW12 & WBLL1/PAURAQ & NIL & $\operatorname{Sr} 30$ & - & - & - & + & - & - \\
\hline 25:ZWW12 & ASTREB/CHONTE & $N I L$ & $\begin{array}{l}\operatorname{Sr} 8 a, \operatorname{Sr} 17 \\
\operatorname{Sr} 30\end{array}$ & - & - & - & - & - & - \\
\hline 26:ZWW12 & $\begin{array}{l}\text { MILAN/KAUZ//DHARWAR } \\
\text { DRY/3/BAV92/4/CHONTE }\end{array}$ & $\operatorname{Yr} 17$ & $\begin{array}{l}\operatorname{Sr} 12, \operatorname{Sr} 17 \\
\operatorname{Sr} 30, \operatorname{Sr} 38\end{array}$ & - & - & - & 1 & + & - \\
\hline
\end{tabular}




\begin{tabular}{|c|c|c|c|c|c|c|c|c|c|}
\hline \multirow[t]{2}{*}{ QCode } & \multirow[t]{2}{*}{ Pedigrees } & \multicolumn{2}{|c|}{ Genes postulated } & \multirow{2}{*}{$\begin{array}{l}Y r 4 \\
\text { barc7 } \\
5 \\
\end{array}$} & \multirow{2}{*}{ 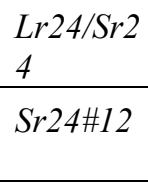 } & \multirow{2}{*}{$\begin{array}{l}\text { Lr26/Yr } \\
9 / \mathrm{Sr} 31 \\
\text { iag95 }\end{array}$} & \multirow{2}{*}{$\begin{array}{l}\text { Lr34/Yr1 } \\
8 / \operatorname{Sr} 57 \\
\operatorname{csLV} 34\end{array}$} & \multirow{2}{*}{$\begin{array}{l}\text { Lr37/Yr17/Sr } \\
38 \\
\text { Ventriup }+L N \\
2\end{array}$} & \multirow{2}{*}{$\begin{array}{l}S r 2 \\
c s S r \\
2 \\
\end{array}$} \\
\hline & & $\begin{array}{l}\text { Stripe } \\
\text { rust }\end{array}$ & Stem rust & & & & & & \\
\hline 28:ZWW12 & $\begin{array}{l}\text { MILAN/KAUZ//DHARWAR } \\
\text { DRY/3/BAV92/4/CHONTE }\end{array}$ & $\operatorname{Yr} 17$ & $\begin{array}{l}\operatorname{Sr} 17, \operatorname{Sr} 30 \\
\operatorname{Sr} 38\end{array}$ & - & - & - & - & + & - \\
\hline 29:ZWW12 & $\begin{array}{l}\text { MILAN/KAUZ//DHARWAR } \\
\text { DRY/3/BAV92/4/PAURAQ }\end{array}$ & $\operatorname{Yr} 6$ & Sr30 & - & - & - & - & - & - \\
\hline 30:ZWW12 & $\begin{array}{l}\text { MILAN/KAUZ//DHARWAR } \\
\text { DRY/3/BAV92/4/PAURAQ }\end{array}$ & $\operatorname{Yr} 17$ & $\begin{array}{l}\text { Sr8a, Sr30, } \\
\operatorname{Sr} 38\end{array}$ & - & - & - & - & + & + \\
\hline 31:ZWW12 & $\begin{array}{l}\text { METSO/ER2000/5/2*SERI*3//RL6010/4*YR/3/PA } \\
\text { STOR/4/BAV92 }\end{array}$ & $\operatorname{Yr} 17$ & $\begin{array}{l}\operatorname{Sr} 17, \operatorname{Sr} 30 \\
\operatorname{Sr} 38\end{array}$ & - & - & - & - & + & - \\
\hline 32:ZWW12 & $\begin{array}{l}\text { METSO/ER2000/5/2*SERI*3//RL6010/4*YR/3/PA } \\
\text { STOR/4/BAV92 }\end{array}$ & $\operatorname{Yr} 17$ & $\begin{array}{l}\text { Sr8a, Sr17, } \\
\operatorname{Sr} 30, \operatorname{Sr} 38\end{array}$ & - & - & - & - & + & - \\
\hline 33:ZWW12 & AGT YOUNG*2//SUNCO/2*PASTOR & $\operatorname{Yr} 17$ & $\operatorname{Sr} 24, \operatorname{Sr} 38$ & - & + & - & + & + & Null \\
\hline 34:ZWW12 & $\begin{array}{l}\text { TOB/ERA//TOB/CNO67/3/PLO/4/VEE\#5/5/KAUZ } \\
\text { /6/FRET2/7/ATTILA*2/PBW65 }\end{array}$ & $Y r ?$ & $\begin{array}{l}\text { Sr } 8 b, \operatorname{Sr} 9 b \\
\operatorname{Sr} 12+\end{array}$ & - & - & - & + & - & - \\
\hline 35:ZWW12 & $\begin{array}{l}\text { TOB/ERA//TOB/CNO67/3/PLO/4/VEE\#5/5/KAUZ } \\
\text { /6/FRET2/7/PASTOR//MILAN/KAUZ/3/BAV92 }\end{array}$ & $\begin{array}{l}\text { Yr3 } \\
\text { Yr17 }\end{array}$ & $\begin{array}{l}\operatorname{Sr} 8 a, \operatorname{Sr} 17 \\
\operatorname{Sr} 30, \operatorname{Sr} 38\end{array}$ & - & - & - & + & + & - \\
\hline 36:ZWW12 & $\begin{array}{l}\text { TOB/ERA//TOB/CNO67/3/PLO/4/VEE\#5/5/KAUZ } \\
\text { /6/FRET2/7/PASTOR//MILAN/KAUZ/3/BAV92 }\end{array}$ & $\operatorname{Yr} 17$ & $\operatorname{Sr} 38+$ & - & - & - & + & + & - \\
\hline 37:ZWW12 & $\begin{array}{l}\text { TOB/ERA//TOB/CNO67/3/PLO/4/VEE\#5/5/KAUZ } \\
\text { /6/FRET2/7/PASTOR//MILAN/KAUZ/3/BAV92 }\end{array}$ & Yr6 & $S r 9 b$ & - & - & - & - & - & - \\
\hline 39:ZWW12 & $\begin{array}{l}\text { TOB/ERA//TOB/CNO67/3/PLO/4/VEE\#5/5/KAUZ } \\
\text { /6/FRET2/7/DANPHE \#1 }\end{array}$ & $\operatorname{Yr} 27$ & Sr17, Sr30 & - & - & - & 1 & - & - \\
\hline 42:ZWW12 & WORRAKATTA/2*PASTOR//DANPHE \# 1 & Yr3, ? & $\operatorname{Sr} 24$ & - & + & - & 1 & - & - \\
\hline
\end{tabular}




\begin{tabular}{|c|c|c|c|c|c|c|c|c|c|}
\hline \multirow[t]{2}{*}{ QCode } & \multirow[t]{2}{*}{ Pedigrees } & \multicolumn{2}{|c|}{ Genes postulated } & \multirow{2}{*}{$\begin{array}{l}Y r 4 \\
\operatorname{barc7} \\
5\end{array}$} & \multirow{2}{*}{$\begin{array}{l}\operatorname{Lr} 24 / S r 2 \\
4 \\
\operatorname{Sr} 24 \# 12\end{array}$} & \multirow{2}{*}{$\begin{array}{l}\text { Lr26/Yr } \\
9 / \mathrm{Sr} 31 \\
\text { iag95 }\end{array}$} & \multirow{2}{*}{$\begin{array}{l}\operatorname{Lr} 34 / \mathrm{Yr} 1 \\
8 / \operatorname{Sr} 57 \\
\operatorname{csLV} 34\end{array}$} & \multirow{2}{*}{$\begin{array}{l}\text { Lr37/Yr17/Sr } \\
38 \\
\text { Ventriup }+L N \\
2\end{array}$} & \multirow{2}{*}{$\begin{array}{l}S r 2 \\
\operatorname{csS} S \\
2\end{array}$} \\
\hline & & $\begin{array}{l}\text { Stripe } \\
\text { rust }\end{array}$ & Stem rust & & & & & & \\
\hline 43:ZWW12 & WORRAKATTA/2*PASTOR//DANPHE \#1 & NIL & $\operatorname{Sr} 23, \operatorname{Sr} 30$ & - & - & - & - & - & - \\
\hline 44:ZWW12 & KRICHAUFF/2*PASTOR//CHONTE & Yr3, ? & $\operatorname{Sr} 24$ & - & + & - & - & - & - \\
\hline 45:ZWW12 & SUNCO.6/FRAME//PASTOR/3/DANPHE \#1 & $\operatorname{Yr} 3, ?$ & $\mathrm{Sr} 30+$ & - & - & - & + & - & - \\
\hline 46:ZWW12 & $\mathrm{KA} / \mathrm{NAC} / / \mathrm{TRCH} / 3 / \mathrm{DANPHE} \# 1$ & NIL & $\operatorname{Sr} 17, \operatorname{Sr} 30$ & - & - & - & - & - & - \\
\hline 48:ZWW12 & $\mathrm{KA} / \mathrm{NAC} / / \mathrm{TRCH} / 3 / \mathrm{DANPHE} \# 1$ & NIL & $\operatorname{Sr} 30$ & - & - & - & - & - & - \\
\hline 49:ZWW12 & KA/NAC//TRCH/3/DANPHE \#1 & NIL & $\mathrm{Sr} 30+$ & - & - & - & - & - & - \\
\hline 51:ZWW12 & BERKUT/MUU//DANPHE \#1 & NIL & $\begin{array}{l}\operatorname{Sr} 17, \operatorname{Sr} 8 b, \\
\operatorname{Sr} 9 b\end{array}$ & - & - & - & 1 & - & - \\
\hline 52:ZWW12 & $\begin{array}{l}\text { AGT } \\
\text { YOUNG*2/5/TUI//2*SUNCO/SA1166/3/TUI/4/FI } \\
\text { NSI }\end{array}$ & $\operatorname{Yr} 6$ & $\begin{array}{l}\operatorname{Sr} 8 a, \operatorname{Sr} 17 \\
\operatorname{Sr} 38\end{array}$ & - & - & - & - & + & Null \\
\hline 53:ZWW12 & $\begin{array}{l}\text { AGT } \\
\text { YOUNG*2/5/TUI//2*SUNCO/SA1166/3/TUI/4/FI } \\
\text { NSI }\end{array}$ & $N I L$ & $\begin{array}{l}\operatorname{Sr} 8 a, \operatorname{Sr} 17 \\
\operatorname{Sr} 38\end{array}$ & - & - & - & + & + & Null \\
\hline 54:ZWW12 & $\begin{array}{l}\text { AGT } \\
\text { YOUNG*2/5/TUI//2*SUNCO/SA1166/3/TUI/4/FI } \\
\text { NSI }\end{array}$ & $\operatorname{Yr} 17$ & $\begin{array}{l}\operatorname{Sr} 8 a, \operatorname{Sr} 17 \\
\operatorname{Sr} 38\end{array}$ & - & - & - & + & + & Null \\
\hline 55:ZWW12 & $\begin{array}{l}\text { METSO/ER2000//MONARCA } \\
\text { F2007/3/WBLL1*2/KKTS }\end{array}$ & $\operatorname{Yr} 17$ & $\operatorname{Sr} 38+$ & - & - & - & + & + & - \\
\hline 56:ZWW12 & $\begin{array}{l}\text { MON/IMU//ALD/PVN/3/BORL95/4/OASIS/2*BO } \\
\text { RL95/5/EMB16/CBRD//CBRD }\end{array}$ & $\operatorname{Yr} 17$ & $\operatorname{Sr} 31, \operatorname{Sr} 38$ & - & + & - & - & + & Null \\
\hline
\end{tabular}




\begin{tabular}{|c|c|c|c|c|c|c|c|c|c|}
\hline \multirow[t]{2}{*}{ QCode } & \multirow[t]{2}{*}{ Pedigrees } & \multicolumn{2}{|c|}{ Genes postulated } & \multirow{2}{*}{$\begin{array}{l}\text { Yr4 } \\
\operatorname{barc7} \\
5\end{array}$} & \multirow{2}{*}{$\begin{array}{l}\operatorname{Lr} 24 / \mathrm{Sr} 2 \\
4 \\
\operatorname{Sr} 24 \# 12\end{array}$} & \multirow{2}{*}{$\begin{array}{l}\text { Lr26/Yr } \\
9 / \mathrm{Sr} 31 \\
\text { iag95 }\end{array}$} & \multirow{2}{*}{$\begin{array}{l}\operatorname{Lr} 34 / \mathrm{Yr} 1 \\
8 / \mathrm{Sr} 57 \\
\operatorname{csLV} 34\end{array}$} & \multirow{2}{*}{$\begin{array}{l}\text { Lr37/Yr17/Sr } \\
38 \\
\text { Ventriup }+L N \\
2\end{array}$} & \multirow{2}{*}{$\begin{array}{l}S r 2 \\
c s S r \\
2\end{array}$} \\
\hline & & $\begin{array}{l}\text { Stripe } \\
\text { rust }\end{array}$ & Stem rust & & & & & & \\
\hline 57:ZWW12 & $\begin{array}{l}\text { 1447/PASTOR//KRICHAUFF/5/2*SERI*3//RL601 } \\
\text { 0/4*YR/3/PASTOR/4/BAV92 }\end{array}$ & $\operatorname{Yr} 17$ & $\begin{array}{l}\text { Sr8a, Sr17, } \\
\operatorname{Sr} 38\end{array}$ & - & - & - & - & + & - \\
\hline 58:ZWW12 & $\begin{array}{l}\text { 1447/PASTOR//KRICHAUFF/5/2*SERI*3//RL601 } \\
\text { 0/4*YR/3/PASTOR/4/BAV92 }\end{array}$ & $\operatorname{Yr} 17$ & $\begin{array}{l}\operatorname{Sr} 8 a, \operatorname{Sr} 17 \\
\operatorname{Sr} 38\end{array}$ & - & - & - & + & + & - \\
\hline 59:ZWW12 & $\begin{array}{l}\text { TUI//2*SUNCO/SA } 1166 / 3 / \text { TUI/4/FINSI/5/SOKOL } \\
\text { L/6/KA/NAC//TRCH }\end{array}$ & $N I L$ & Sr30 & - & - & - & 1 & - & - \\
\hline 61:ZWW12 & ITP50/3/KA/NAC//TRCH & $Y r ?$ & $\operatorname{Sr} 30$ & - & - & - & 1 & - & - \\
\hline 62:ZWW12 & $\begin{array}{l}\text { EMB16/CBRD//CBRD/3/SUNCO.6/FRAME//PAS } \\
\text { TOR/4/MILAN/KAUZ//DHARWAR } \\
\text { DRY/3/BAV92 }\end{array}$ & $\begin{array}{l}\operatorname{Yr} 9, \\
\operatorname{Yr} 17\end{array}$ & $\begin{array}{l}\text { Sr24, Sr31, } \\
\text { Sr38 }\end{array}$ & - & + & + & + & + & - \\
\hline 63:ZWW12 & $\begin{array}{l}\text { C } 80.1 / 3 * \text { BATAVIA//2*WBLL1/3/EMB16/CBRD// } \\
\text { CBRD/4/MILAN/KAUZ//DHARWAR } \\
\text { DRY/3/BAV92 }\end{array}$ & $\begin{array}{l}\operatorname{Yr} 4 \\
\operatorname{Yr} 9 \\
\operatorname{Yr} 17\end{array}$ & $\operatorname{Sr} 31, \operatorname{Sr} 38$ & + & - & + & 1 & + & Null \\
\hline 64:ZWW12 & $\begin{array}{l}\text { C } 80.1 / 3 * \text { BATAVIA//2*WBLL1/3/EMB16/CBRD// } \\
\text { CBRD/4/MILAN/KAUZ//DHARWAR } \\
\text { DRY/3/BAV92 }\end{array}$ & $\begin{array}{l}\operatorname{Yr} 3 \\
\operatorname{Yr} 9 \\
\operatorname{Yr} 17\end{array}$ & $\operatorname{Sr} 31, \operatorname{Sr} 38$ & - & - & + & - & + & Null \\
\hline 67:ZWW12 & $\begin{array}{l}\mathrm{KA} / \mathrm{NAC} / / \mathrm{TRCH} / 3 / \mathrm{SLVS} / \mathrm{ATTILA} / \mathrm{WBLL} 1 / 4 / \mathrm{KA} \\
/ \mathrm{NAC} / / \mathrm{TRCH}\end{array}$ & NIL & $\operatorname{Sr} 30$ & - & - & - & - & - & - \\
\hline 68:ZWW12 & $\begin{array}{l}\mathrm{KA} / \mathrm{NAC} / / \mathrm{TRCH} / 3 / \mathrm{SLVS} / \mathrm{ATTILA} / \mathrm{WBLL} 1 / 4 / \mathrm{KA} \\
\text { /NAC//TRCH }\end{array}$ & NIL & $S r 8 a, S r 30$ & - & - & - & - & - & - \\
\hline 69:ZWW12 & $\begin{array}{l}\mathrm{KA} / \mathrm{NAC} / / \mathrm{TRCH} / 3 / \mathrm{SLVS} / \mathrm{ATTILA} / / \mathrm{WBLL} 1 / 4 / \mathrm{KA} \\
/ \mathrm{NAC} / / \mathrm{TRCH}\end{array}$ & $N I L$ & Sr8a, Sr30 & - & - & - & - & - & - \\
\hline
\end{tabular}




\begin{tabular}{|c|c|c|c|c|c|c|c|c|c|}
\hline \multirow[t]{2}{*}{ QCode } & \multirow[t]{2}{*}{ Pedigrees } & \multicolumn{2}{|c|}{ Genes postulated } & \multirow{2}{*}{$\begin{array}{l}\text { Yr4 } \\
\operatorname{barc7} \\
5\end{array}$} & \multirow{2}{*}{$\begin{array}{l}\operatorname{Lr} 24 / S r 2 \\
4 \\
\operatorname{Sr} 24 \# 12\end{array}$} & \multirow{2}{*}{$\begin{array}{l}\text { Lr26/Yr } \\
9 / \mathrm{Sr} 31 \\
\text { iag95 }\end{array}$} & \multirow{2}{*}{$\begin{array}{l}\operatorname{Lr} 34 / Y r 1 \\
8 / \operatorname{Sr} 57 \\
\operatorname{csLV} 34\end{array}$} & \multirow{2}{*}{$\begin{array}{l}\text { Lr37/Yr17/Sr } \\
38 \\
\text { Ventriup }+L N \\
2\end{array}$} & \multirow{2}{*}{$\begin{array}{l}S r 2 \\
c s S r \\
2\end{array}$} \\
\hline & & $\begin{array}{l}\text { Stripe } \\
\text { rust }\end{array}$ & Stem rust & & & & & & \\
\hline 70:ZWW12 & $\begin{array}{l}\mathrm{KA} / \mathrm{NAC} / / \mathrm{TRCH} / 3 / \mathrm{SLVS} / \mathrm{ATTILA} / / \mathrm{WBLL} 1 / 4 / \mathrm{KA} \\
\text { /NAC//TRCH }\end{array}$ & $N I L$ & Sr8a, Sr30 & - & - & - & - & - & - \\
\hline 77:ZWW12 & $\begin{array}{l}\text { SUNCO/2*PASTOR/3/SLVS/ATTILA//WBLL1/4/ } \\
\text { KA/NAC//TRCH }\end{array}$ & $N I L$ & Sr30 & - & - & - & - & - & - \\
\hline 78:ZWW12 & $\begin{array}{l}\text { SLVS/3/CROC_1/AE.SQUARROSA } \\
(224) / / \text { OPATA/5/VEE/LIRA//BOW/3/BCN/4/KAU } \\
\text { Z/6/2*KA/NAC//TRCH }\end{array}$ & $N I L$ & Sr30 & - & - & - & - & - & - \\
\hline 85:ZWW12 & $\begin{array}{l}\text { CNDO/R143//ENTE/MEXI_2/3/AEGILOPS } \\
\text { SQUARROSA } \\
\text { (TAUS)/4/WEAVER/5/2*JANZ/6/SERI*3//RL601 } \\
\text { 0/4*YR/3/PASTOR/4/BAV92/7/VORB }\end{array}$ & $\begin{array}{l}\operatorname{Yr} 3, ? \\
\operatorname{Yr} 6\end{array}$ & Sr30 & - & - & - & - & - & - \\
\hline 87:ZWW12 & $\begin{array}{l}\text { CNDO/R143//ENTE/MEXI_2/3/AEGILOPS } \\
\text { SQUARROSA } \\
\text { (TAUS)/4/WEAVER/5/2*JANZ/8/CAL/NH//H567. } \\
\text { 71/3/SERI/4/CAL/NH//H567.71/5/2*KAUZ/6/WH } \\
\text { 576/7/WH 542 }\end{array}$ & $\operatorname{Yr} 27+$ & Sr9b or $\mathrm{Sr} 30$ & - & - & - & - & - & - \\
\hline 88:ZWW12 & $\begin{array}{l}\text { CNDO/R143//ENTE/MEXI_2/3/AEGILOPS } \\
\text { SQUARROSA } \\
\text { (TAUS)/4/WEAVER/5/2*JANZ/6/VORB }\end{array}$ & $\operatorname{Yr} 27$ & $\begin{array}{l}\text { Sr8a,Sr12, } \\
\operatorname{Sr} 30\end{array}$ & - & - & - & + & - & - \\
\hline 97:ZWW12 & $\begin{array}{l}\text { WORRAKATTA/2*PASTOR//PARUS/PASTOR/3 } \\
\text { /SOKOLL }\end{array}$ & $\operatorname{Yr} 17$ & $\begin{array}{l}\text { Sr8a, Sr17, } \\
\operatorname{Sr} 30, \operatorname{Sr} 38\end{array}$ & - & - & - & + & + & Null \\
\hline 98:ZWW12 & $\begin{array}{l}\text { TOB/ERA//TOB/CNO67/3/PLO/4/VEE\#5/5/KAUZ } \\
\text { /6/FRET2/7/VORB }\end{array}$ & Yr6 & $\mathrm{Sr} 8 a, \mathrm{Sr} 30$ & - & - & - & + & - & - \\
\hline
\end{tabular}




\begin{tabular}{|c|c|c|c|c|c|c|c|c|c|}
\hline \multirow[t]{2}{*}{ QCode } & \multirow[t]{2}{*}{ Pedigrees } & \multicolumn{2}{|c|}{ Genes postulated } & \multirow{2}{*}{$\begin{array}{l}\text { Yr4 } \\
\operatorname{barc7} \\
5\end{array}$} & \multirow{2}{*}{$\begin{array}{l}\operatorname{Lr} 24 / S r 2 \\
4 \\
S r 24 \# 12\end{array}$} & \multirow{2}{*}{$\begin{array}{l}\text { Lr26/Yr } \\
9 / \mathrm{Sr} 31 \\
\text { iag95 }\end{array}$} & \multirow{2}{*}{ 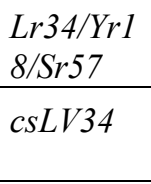 } & \multirow{2}{*}{$\begin{array}{l}\text { Lr37/Yr17/Sr } \\
38 \\
\text { Ventriup }+L N \\
2\end{array}$} & \multirow{2}{*}{$\begin{array}{l}S r 2 \\
\operatorname{csS} r \\
2\end{array}$} \\
\hline & & $\begin{array}{l}\text { Stripe } \\
\text { rust }\end{array}$ & Stem rust & & & & & & \\
\hline 100:ZWW12 & $\begin{array}{l}\text { TOB/ERA//TOB/CNO67/3/PLO/4/VEE\#5/5/KAUZ } \\
\text { /6/FRET2/7/PASTOR//MILAN/KAUZ/3/BAV92 }\end{array}$ & $\operatorname{Yr} 17$ & Sr38+ & - & - & - & - & + & - \\
\hline 101:ZWW12 & $\begin{array}{l}\text { TOB/ERA//TOB/CNO67/3/PLO/4/VEE\#5/5/KAUZ } \\
\text { /6/FRET2/7/PASTOR//MILAN/KAUZ/3/BAV92 }\end{array}$ & $\operatorname{Yr} 6$ & $\begin{array}{l}\operatorname{Sr} 8 a, \operatorname{Sr} 12 \\
\operatorname{Sr} 30\end{array}$ & - & - & - & - & - & - \\
\hline 102:ZWW12 & $\begin{array}{l}\text { TOB/ERA//TOB/CNO67/3/PLO/4/VEE\#5/5/KAUZ } \\
\text { /6/FRET2/7/PASTOR//MILAN/KAUZ/3/BAV92 }\end{array}$ & NIL & $\mathrm{Sr} 30+$ & - & - & - & - & - & - \\
\hline 107:ZWW12 & $\begin{array}{l}\text { TOB/ERA//TOB/CNO67/3/PLO/4/VEE\#5/5/KAUZ } \\
\text { /6/FRET2/7/PAURAQ }\end{array}$ & $Y r, ?$ & Sr8a, Sr30 & - & - & - & + & - & - \\
\hline 108:ZWW12 & $\begin{array}{l}\text { NS- } \\
\text { 732/HER/3/PRL/SARA//TSI/VEE\#5/4/FRET2/5/C } \\
\text { HONTE }\end{array}$ & $\operatorname{Yr} 27$ & Sr8a, Sr30 & - & - & - & - & - & - \\
\hline 109:ZWW12 & KA/NAC//TRCH/3/VORB & $\operatorname{Yr} 6$ & Sr30 & - & - & - & 1 & - & - \\
\hline 111:ZWW12 & $\begin{array}{l}\text { KA/NAC//TRCH/4/MILAN/KAUZ//DHARWAR } \\
\text { DRY/3/BAV92 }\end{array}$ & Yr3,?+ & $\begin{array}{l}\text { Sr8b+??, } \\
\text { Sr30 }\end{array}$ & - & - & - & - & - & - \\
\hline 112:ZWW12 & $\mathrm{KA} / \mathrm{NAC} / / \mathrm{TRCH} / 3 / \mathrm{DANPHE} \# 1$ & $\begin{array}{l}\text { Yr34,? } \\
+\end{array}$ & $\begin{array}{l}\operatorname{Sr} 8 b, \operatorname{Sr} 12 \\
\operatorname{Sr} 30\end{array}$ & - & - & - & - & - & - \\
\hline 113:ZWW12 & $\mathrm{KA} / \mathrm{NAC} / / \mathrm{TRCH} / 3 / \mathrm{DANPHE} \# 1$ & $Y r 3, ?$ & $\begin{array}{l}\text { Sr8b, Sr12, } \\
\operatorname{Sr} 30\end{array}$ & - & - & - & - & - & - \\
\hline 114:ZWW12 & KA/NAC//TRCH/3/DANPHE \#1 & Yr34,? & $\operatorname{Sr} 8 b, \operatorname{Sr} 12$ & - & - & - & - & - & - \\
\hline 116:ZWW12 & KA/NAC//TRCH/3/DANPHE \#1 & Yr34? & $S r 8 b$ & - & - & - & - & - & - \\
\hline 117:ZWW12 & $\mathrm{KA} / \mathrm{NAC} / / \mathrm{TRCH} / 3 / \mathrm{DANPHE} \# 1$ & Yr34, ? & $\operatorname{Sr} 8 b, S r 9 b$ & - & - & - & 1 & - & - \\
\hline
\end{tabular}




\begin{tabular}{|c|c|c|c|c|c|c|c|c|c|}
\hline \multirow[t]{2}{*}{ QCode } & \multirow[t]{2}{*}{ Pedigrees } & \multicolumn{2}{|c|}{ Genes postulated } & \multirow{2}{*}{$\begin{array}{l}Y r 4 \\
\text { barc7 } \\
5 \\
\end{array}$} & \multirow{2}{*}{$\begin{array}{l}L r 24 / S r 2 \\
4 \\
S r 24 \# 12\end{array}$} & \multirow{2}{*}{$\begin{array}{l}\text { Lr26/Yr } \\
\text { 9/Sr31 } \\
\text { iag95 }\end{array}$} & \multirow{2}{*}{$\begin{array}{l}\text { Lr34/Yr1 } \\
8 / S r 57 \\
c s L V 34\end{array}$} & \multirow{2}{*}{$\begin{array}{l}\text { Lr37/Yr17/Sr } \\
38 \\
\text { Ventriup }+L N \\
2\end{array}$} & \multirow{2}{*}{$\begin{array}{l}S r 2 \\
c s S r \\
2\end{array}$} \\
\hline & & $\begin{array}{l}\text { Stripe } \\
\text { rust }\end{array}$ & Stem rust & & & & & & \\
\hline 118:ZWW12 & $\mathrm{KA} / \mathrm{NAC} / / \mathrm{TRCH} / 3 / \mathrm{DANPHE} \# 1$ & $Y r ?$ & Sr30 & - & - & - & - & - & - \\
\hline 119:ZWW12 & $\mathrm{KA} / \mathrm{NAC} / / \mathrm{TRCH} / 3 / \mathrm{DANPHE} \# 1$ & $\operatorname{Yr} 3, ?$ & $S r 9 b, S r 8 b$ & - & - & - & - & - & - \\
\hline 120:ZWW12 & $\mathrm{KA} / \mathrm{NAC} / / \mathrm{TRCH} / 3 / \mathrm{DANPHE} \# 1$ & NIL & $\begin{array}{l}\text { Sr } 8 b, \operatorname{Sr} 9 b \\
\operatorname{Sr} 12\end{array}$ & - & - & - & - & - & - \\
\hline 122:ZWW12 & KA/NAC//TRCH/3/PAURAQ & $N I L$ & $S r 9 b$ & - & - & - & + & - & - \\
\hline 126:ZWW12 & $\begin{array}{l}\mathrm{KA} / \mathrm{NAC} / / \mathrm{TRCH} / 5 / \mathrm{SERI} \text { *3//RL6010/4*YR/3/PAS } \\
\text { TOR/4/BAV92 }\end{array}$ & NIL & $\operatorname{Sr} 30$ & - & - & - & - & - & - \\
\hline 127:ZWW12 & $\mathrm{KA} / \mathrm{NAC} / / \mathrm{TRCH} / 3 / \mathrm{KINDE}$ & $\operatorname{Yr} 17$ & $\operatorname{Sr} 38+$ & - & - & - & - & + & - \\
\hline 128:ZWW12 & $\begin{array}{l}\text { MILAN/KAUZ//DHARWAR } \\
\text { DRY/3/BAV92/4/CHONTE }\end{array}$ & $\operatorname{Yr} 17$ & $\operatorname{Sr} 38+$ & - & - & - & - & + & - \\
\hline 129:ZWW12 & $\begin{array}{l}\text { MILAN/KAUZ//DHARWAR } \\
\text { DRY/3/BAV92/4/CHONTE }\end{array}$ & NIL & $\operatorname{Sr} 24+$ & - & + & - & - & - & - \\
\hline 130:ZWW12 & $\begin{array}{l}\text { WORRAKATTA/2*PASTOR//MUU/3/DANPHE } \\
\# 1\end{array}$ & $\operatorname{Yr} 17$ & Sr30, Sr38+ & - & - & - & + & + & - \\
\hline 131:ZWW12 & $\begin{array}{l}\text { MON/IMU//ALD/PVN/3/BORL95/4/OASIS/2*BO } \\
\text { RL95/5/2*SKAUZ/BAV92 }\end{array}$ & Yr3, ? & $\begin{array}{l}\text { Sr8a, } \operatorname{Sr} 17 \\
\operatorname{Sr} 30\end{array}$ & - & - & - & - & - & + \\
\hline 132:ZWW12 & BERKUT/MUU//MUU & NIL & $\operatorname{Sr} 17, \operatorname{Sr} 30$ & - & - & - & + & - & + \\
\hline 134:ZWW12 & BERKUT/MUU//DANPHE \#1 & NIL & $\operatorname{Sr} 17, \operatorname{Sr} 30+$ & - & + & - & + & - & + \\
\hline 136:ZWW12 & $\begin{array}{l}\text { EMB16/CBRD//CBRD/4/BETTY/3/CHEN/AE.SQ/ } \\
/ 2 * \text { OPATA }\end{array}$ & $\operatorname{Yr} 27$ & $\operatorname{Sr} 17, \operatorname{Sr} 30$ & - & - & - & - & - & - \\
\hline
\end{tabular}




\begin{tabular}{|c|c|c|c|c|c|c|c|c|c|}
\hline \multirow[t]{2}{*}{ QCode } & \multirow[t]{2}{*}{ Pedigrees } & \multicolumn{2}{|c|}{ Genes postulated } & \multirow{2}{*}{$\begin{array}{l}Y r 4 \\
\operatorname{barc7} \\
5\end{array}$} & \multirow{2}{*}{$\begin{array}{l}\operatorname{Lr} 24 / \mathrm{Sr} 2 \\
4 \\
\operatorname{Sr} 24 \# 12\end{array}$} & \multirow{2}{*}{$\begin{array}{l}\text { Lr26/Yr } \\
9 / \mathrm{Sr} 31 \\
\text { iag95 }\end{array}$} & \multirow{2}{*}{$\begin{array}{l}\operatorname{Lr} 34 / Y r 1 \\
8 / \operatorname{Sr} 57 \\
\operatorname{csLV} 34\end{array}$} & \multirow{2}{*}{$\begin{array}{l}\text { Lr37/Yr17/Sr } \\
38 \\
\text { Ventriup }+L N \\
2\end{array}$} & \multirow{2}{*}{$\begin{array}{l}S r 2 \\
\operatorname{csSr} \\
2 \\
\end{array}$} \\
\hline & & $\begin{array}{l}\text { Stripe } \\
\text { rust }\end{array}$ & Stem rust & & & & & & \\
\hline 137:ZWW12 & $\begin{array}{l}\text { PASTOR//HXL7573/2*BAU*2/3/PFAU/WEAVER } \\
\text { //KIRITATI }\end{array}$ & $N I L$ & $\operatorname{Sr} 17, \operatorname{Sr} 24$ & - & + & - & - & - & - \\
\hline 138:ZWW12 & $\begin{array}{l}\text { CNDO/R143//ENTE/MEXI_2/3/AEGILOPS } \\
\text { SQUARROSA } \\
\text { (TAUS)/4/WEAVER/5/2*JANZ/6/SKAUZ/BAV92 }\end{array}$ & $\operatorname{Yr} 17$ & $\begin{array}{l}\text { Sr8a, Sr9b, } \\
\text { Sr17, Sr38 }\end{array}$ & - & - & - & - & + & - \\
\hline 140:ZWW12 & METSO/ER2000/3/PASTOR//HXL7573/2*BAU & $\operatorname{Yr} 17$ & $\operatorname{Sr} 38+$ & - & - & - & - & + & - \\
\hline 141:ZWW12 & METSO/ER2000/3/PASTOR//HXL7573/2*BAU & $\operatorname{Yr} 17$ & $\operatorname{Sr} 38+$ & - & - & - & - & + & - \\
\hline 142:ZWW12 & METSO/ER2000/3/PASTOR//HXL7573/2*BAU & $\operatorname{Yr} 17$ & $\operatorname{Sr} 38+$ & - & - & - & - & + & - \\
\hline 145:ZWW12 & METSO/ER2000//PBW343*2/KUKUNA & $\operatorname{Yr} 27$ & Sr8a, Sr30 & - & - & - & - & - & Null \\
\hline
\end{tabular}


Table 2. List of P. graminis f. sp. tritici (Pgt) and P. striiformis f. sp. tritici (Pst) pathotypes used and their virulence spectrum

\begin{tabular}{|c|c|c|c|c|}
\hline PBIC & Pathotype & PBIC & Avirulence & Virulence \\
\hline & Pgt & & & \\
\hline 103 & $34-1,2,3,4,5,6,7$ & 74-L-1 & Sr $8 b, 9 e, 13,24,27,30,32,33,35,37,38,39,40,45,46$, Agi,Em,satu & $\operatorname{Sr} 5,6,8 a, 9 b, 9 g, 11,12,15,17,36$ \\
\hline 205 & $34-1,2,3,6,7,(8), 9$ & 76-L-7 & Sr $8 b, 9 e, 13,17,24,27,32,33,35,36,37,38,39,40,45,46$, Em,satu & $\operatorname{Sr} 5,6,8 a, 9 b, 9 g, 11,12,15,30, A g i$ \\
\hline 206 & $34-1,2,3,5,7,8,9$ & $76-\mathrm{L}-8$ & Sr $8 a, 8 b, 9 e, 13,24,27,32,33,35,36,37,38,39,40,45,46$, Em,satu & $\operatorname{Sr} 5,9 b, 9 g, 11,12,15,17,30, A g i$ \\
\hline 465 & $343-1,2,3,5,6,8,9$ & 890005 & Sr8b,9e,9g,13,15,24,27,32,33,35,36,37,38,39,40,45,46, Em,satu & $\operatorname{Sr} 5,6,8 a, 9 b, 11,12,17,(30), A g i$ \\
\hline 279 & $98-1,2,(3),(5), 6$ & 780129 & $\begin{array}{l}\text { Sr8b,9e,13,15,24,27,30,32,33,35,36,37,38,39,40,45,46, } \\
\text { Agi,Em,satu }\end{array}$ & $\operatorname{Sr} 5,6,8 a,(9 b), 9 g, 11,12,(17)$ \\
\hline 565 & $34-1,2,7+\operatorname{Sr} 38$ & 10130 & $\begin{array}{l}\text { Sr8a,8b,9b,9e,13,17,24,27,30,32,33,35,36,37,39,40,45,46, } \\
\text { Agi,Em,satu }\end{array}$ & $\operatorname{Sr} 5,6,7 b, 9 g, 11,15,38$ \\
\hline \multirow[t]{2}{*}{99} & $34-2,4,5,7,11$ & 640231 & Sr $8 a, 9 b, 9 e, 13,24,27,30,32,33,35,37,38,39,40,45,46$, Agi,Em,satu & $\operatorname{Sr} 5,6,7 b, 9 g, 11,15,17,36$ \\
\hline & Pst & & & \\
\hline 599 & $134 \mathrm{E} 16 \mathrm{~A}+\operatorname{Yr} 17+$ & 61639 & $\operatorname{Yr} 1,3,4,5,10,15,24,27,32,33,34,47, S D, S u, N D, S p$ & $\operatorname{Yr} 2,6,7,8,9,17, A$ \\
\hline 617 & $\begin{array}{l}\text { 134E16A+Yr17+ } \\
Y r 27+\end{array}$ & 101975 & $\operatorname{Yr} 1,3,4,5,10,15,24,32,33,34,47, S D, S u, N D, S p$ & $\operatorname{Yr} 2,6,7,8,9,17, A, 27$ \\
\hline 444 & $110 \mathrm{E} 143 \mathrm{~A}+$ & 861725 & $\operatorname{Yr} 1,5,8,9,10,15,17,24,27,32,33,47, S p$ & $\operatorname{Yr} 2,3,4,6,7, S D, S u, N D, A, 34$ \\
\hline 420 & $108 \mathrm{E} 141 \mathrm{~A}^{+}$ & 831917 & $\operatorname{Yr} 1,5,7,8,9,10,15,17,24,27,32,33,47, S p$ & $\operatorname{Yr2,3,4,6,A,SD,Su,ND,34}$ \\
\hline 414 & 104E137A+ + & 821552 & $\operatorname{Yr} 1,5,6,7,8,9,10,27,32,33,47, S p$ & $Y r 2,3,4, A, 34$ \\
\hline
\end{tabular}

PBIC: Plant Breeding Institute culture number assigned in the cereal rust collection 


\section{Greenhouse tests}

Experimental materials were sown in $9 \mathrm{~cm}$ diameter plastic pots filled with a mixture of pine bark and river sand in a ratio of 2:1. An initial dose of $10 \mathrm{~g}$ of water-soluble fertilizer Aquasol ${ }^{\circledR}$ dissolved in 10 litres of tap water was applied to the filled pots before sowing. Four entries were sown per pot as 10 seeds clump. Seven-day old seedlings were fertilized with Urea at the same dose as Aquasol®. Ten to 12-day old seedlings were inoculated with urediniospores of the different Pgt and Pst pathotypes suspended in light mineral oil Isopar- $\mathrm{L}^{\circledR}$ using a hydrocarbon pressure pack. Stem rust inoculated seedlings were humidified on water filled steel trays covered with plastic hoods under natural light at $18-20^{\circ} \mathrm{C}$ for 48 hours, while stripe rust inoculated seedlings were incubated in the dark at $9 \pm 2^{\circ} \mathrm{C}$ for 24 hours. Following incubation, seedlings were moved to microclimate rooms maintained at $25 \pm 2^{\circ} \mathrm{C}$ (stem rust) and at $17 \pm 2^{\circ} \mathrm{C}$ (stripe rust). Two sets were planted for screening against Pgt pathotype 34-2,4,5,7,11. One set each was incubated at $25 \pm 2{ }^{\circ} \mathrm{C}$ and $20 \pm 2^{\circ} \mathrm{C}$ post inoculation to enable postulation of temperaturesensitive stem rust resistance genes Sr6 and Sr12 (Tsilo et al., 2009). Seedling stem rust assessments were made 14 days after inoculation using the 0-4 scale described in McIntosh $e t$ al., (1995), while stripe rust responses were scored 15 days post inoculation using 0-4 scale as described in Bariana and McIntosh, (1993). The description of infection types (ITs) used to classify the reactions to stem rust are: 0 (no visible uredia); (hypersensitive flecks), 1 (small uredia with necrosis), 2 (small to medium sized uredia with green islands surrounded by necrosis and chlorosis), 3 (medium sized uredia with or without chlorosis), 4 (large uredia without chlorosis) and X (heterogenous, similarly distributed all over the leaves). Stripe rust ITs include: 0 (no visible uredia); (necrotic flecks); $\mathrm{N}$ (necrotic areas without sporulation), 1 (necrotic and chlorotic areas with restricted sporulation), 2 (moderate sporulation with necrosis and chlorosis), 3 (sporulation with chlorosis) and 4 (abundant sporulation without chlorosis). Infection types 0 to 2 were considered to show avirulence (low infection) for a particular $\mathrm{Sr}$ gene and infection types 3 to 4 denoted virulence (high infection). Tests were repeated to clarify ambiguous results.

\section{DNA extraction and quantification}

Leaf samples of $2 \mathrm{~cm}$ length from leaves (2-leaf stage) of each entry were collected in $2 \mathrm{ml}$ tubes and dried on silica gel for 3 days. DNA was extracted from the 95 wheat entries following the procedure described in Bansal et al., (2014). DNA was quantified using a nanodrop ND-100 spectrophotometer and dilutions of $30 \mathrm{ng} / \mu \mathrm{l}$ of genomic DNA were made using deionized water.

\section{Molecular marker genotyping}

The entire wheat nursery was genotyped with gene-linked markers to detect the presence of

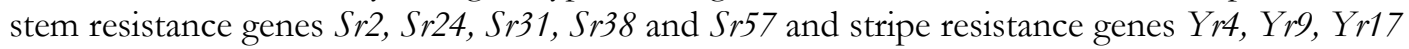
and Yr18 (Table 3). Hartog (Sr2), Janz (Sr24, Yr18/Sr57), Sunlin (Sr26), AvS/6*Yr9 (Sr31/Yr9), Trident (Sr38/Yr17) and Rubric (Yr4) were included as positive controls for the respective markers. 
Table 3. Details of markers used

\begin{tabular}{|c|c|c|c|c|c|}
\hline Gene & Marker & Amplicon (bp) & Forward primer sequence & Reverse primer Sequence & Reference \\
\hline Yr4 & barc75 & $\begin{array}{l}Y r 4 Y r 4=132 \\
y r 4 y r 4=137 \\
\text { and } 139\end{array}$ & AGGGTTACAGTTTGCTCTTTTAC & $\begin{array}{l}\text { CCCGACGACCTATCTATACTTCTC } \\
\text { TA }\end{array}$ & $\begin{array}{l}\text { Somers et } \\
\text { al. }(2004)\end{array}$ \\
\hline$L r 24 / S r 24$ & $\operatorname{Sr} 24 \# 12$ & $\begin{array}{l}S r 24 S r 24=500 \\
s r 24 s r 24=\text { null }\end{array}$ & CAC CCG TGA CAT GCT CGT A & $\begin{array}{l}\text { AAC AGG AAA TGA GCA ACG ATG } \\
T\end{array}$ & $\begin{array}{l}\text { Mago et al. } \\
(2005)\end{array}$ \\
\hline $\begin{array}{l}\operatorname{Lr} 26 / Y r 9 / S \\
r 31\end{array}$ & iag95 & $\begin{array}{l}\operatorname{Yr} 9 Y r 9=1100 \\
y r 9 y r 9=\text { null }\end{array}$ & $\begin{array}{l}\text { CTCTGTGGATAGTTACTTGATCG } \\
\text { A }\end{array}$ & CCTAGAACATGCATGGCTGTTACA & $\begin{array}{l}\text { Mago et al. } \\
(2007)\end{array}$ \\
\hline $\operatorname{Lr} 34 / Y r 18$ & $\operatorname{csLV} 34$ & $\begin{array}{l}\operatorname{Lr} 34 \operatorname{Lr} 34=150 \\
\operatorname{lr} 34 \operatorname{lr} 34=229\end{array}$ & $\begin{array}{l}\text { GTTGGTTAAGACTGGTGGTGAT } \\
\text { GG }\end{array}$ & TGCTTGCTATTGCTGCTGAATAGT & $\begin{array}{l}\text { Lagudah et } \\
\text { al. (2007) }\end{array}$ \\
\hline $\begin{array}{l}\operatorname{Lr} 37 / Y r 17 / \\
\operatorname{Sr} 38\end{array}$ & $\begin{array}{l}\text { Ventriup }+ \\
\text { LN2 }\end{array}$ & $\begin{array}{l}\operatorname{Yr} 17 \operatorname{Yr} 17=262 \\
\text { yr } 17 y r 17=\text { null }\end{array}$ & AGGGGCTACTGACCAAGGCT & $\begin{array}{l}\text { TGCAGCTACAGCAGTATGTACACA } \\
\text { AAA }\end{array}$ & $\begin{array}{l}\text { Helguera et } \\
\text { al. (2007) }\end{array}$ \\
\hline $\operatorname{Sr} 2$ & $\operatorname{csS} 2$ & $\begin{array}{l}\operatorname{Sr} 2 S r 2=337 \\
s r 2 s r 2=\text { null }\end{array}$ & $\begin{array}{l}\text { CAA GGG TTG CTA GGA TTG } \\
\text { GAA AAC }\end{array}$ & $\begin{array}{l}\text { AGA TAA CTC TTA TGA TCT TAC } \\
\text { ATT TTT CTG }\end{array}$ & $\begin{array}{l}\text { Mago et al. } \\
\text { (2010) }\end{array}$ \\
\hline
\end{tabular}


PCR amplifications were performed at the University of Sydney Plant Breeding Institute, Cobbiity, Australia in $10 \mu \mathrm{l}$ reaction volumes containing $60 \mathrm{ng} / \mu \mathrm{l}$ of genomic DNA from each entry and respective controls, $0.2 \mathrm{mM}$ dNTPs, $1 \times$ PCR buffer containing $1.5 \mathrm{mM} \mathrm{MgCl} 2$ (Bioline), $0.5 \mu \mathrm{M}$ of each primer (forward and reverse) and $0.02 \mathrm{U}$ Immolase Taq DNA polymerase (Bioline). PCR reactions were performed in T100TM thermal cycler (BioRad USA) using published PCR conditions/profiles for the different primers. PCR products and restriction enzyme digests (csSr2) were separated on $2 \%$ agarose gels stained with gel red and visualised under UV gel documentation system (UVP-GelDoc-It). PCR amplification for $\mathrm{M}_{13^{-}}$ labelled barc75 was carried out in a total reaction volume of $10 \mu \mathrm{l}$ containing $30 \mathrm{ng} / \mu \mathrm{l}$ of genomic DNA, $1 \times \mathrm{MgCl}_{2}$ buffer, $0.75 \times \mathrm{dNTPs}, 0.4 \times 1.25 \mu \mathrm{M}$ forward primer labelled with $\mathrm{M}_{13}$, $0.4 \times 5 \mu \mathrm{M}$ Reverse primer, $0.1 \times 0.50 \mu \mathrm{M} \mathrm{M}_{13}$-tailed primer labelled with IRDye 700 or 800 and $0.04 \mu \mathrm{l} \times 0.02 \mathrm{U}$ Immolase Taq DNA polymerase (Bioline). The PCR reactions were carried out in BioRad machine and PCR products were separated on 6.5\% Polyacrylamide gel using electrophoresis apparatus LICOR-4300 DNA analyser system (Li-COR Bio-science USA). GeneRuler ${ }^{\mathrm{TM}} 1 \mathrm{~Kb}$ ladder (Fermentas) was used to determine allele sizes.

\section{Results}

\section{Postulation of resistance genes}

Resistance genes were postulated by comparing infection types (ITs) of the different Pgt and Pst pathotypes on test genotypes with those of differential lines with known resistance genes. A high IT (3-4) on a test genotype demonstrated the lack of resistance gene for which that pathotype was avirulent and the differential stock for the target gene also expressed high IT. Genotypes that show low ITs (0-2) with all pathotypes are likely to carry either a gene effective against all pathotypes or combinations of genes with compensating pathotypic specificities. Fig. 1 illustrates the various rust responses observed among the wheat screening nursery tested.

a

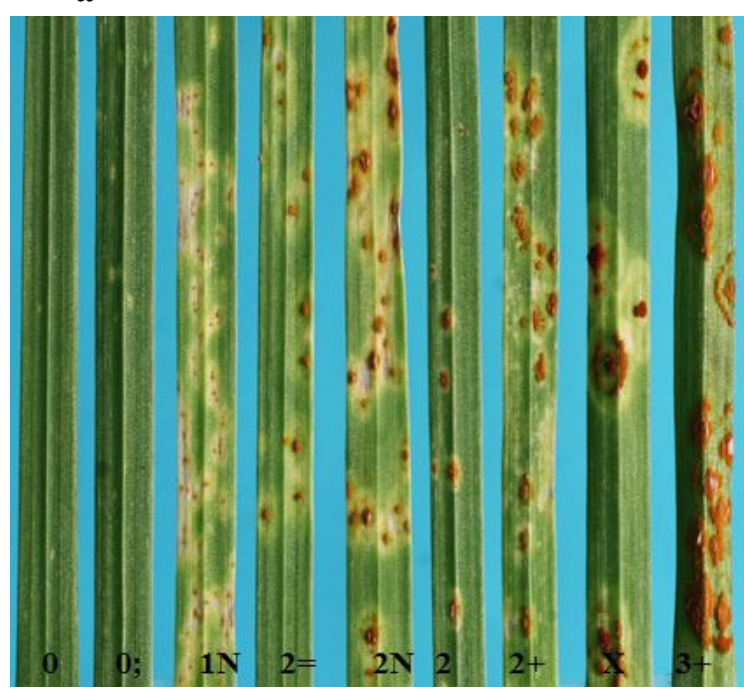

b

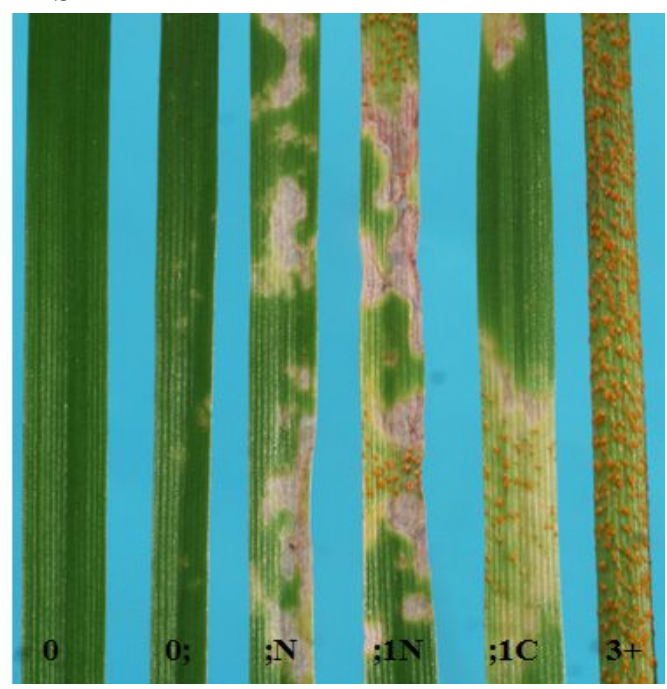

Fig. 1 a) Seedling stem rust b) seedling stripe rust response variations observed among the 95 entries tested in this international wheat nursery. 


\section{Stem rust}

The performance of the 95 wheat lines tested against the seven pathotypes is summarized in Table 4 . Seventy-three $(76.8 \%)$ of the tested entries showed varying levels of resistance (ITs 0 , ; ; ; 1 1C, and 2) to races 34-1,2,3,4,5,6,7 (103); 34-1,2,3,6,7,(8),9 (205); 34-1,2,3,5,7,8,9 (206); 343-1,2,3,5,6,(8),9 (465); 98-1,2,(3),(5),6 (279); 34-1,2,7+Sr38 (565); 34-2,4,5,7,11 (99). The remaining $23.2 \%$ showed varying levels of susceptibility (ITs 3, 3-, 3+, and 4) Seedling stem rust resistance genes $\mathrm{Sr} 8$ a, Sr8b, Sr9b, Sr12, Sr17, Sr23, Sr24, Sr30, Sr31 and Sr38 were postulated (Table 1 and Table 5). Majority of these genes were present in combinations and a few lines carried $\operatorname{Sr} 8 a(1), \operatorname{Sr} 8 b(1), \operatorname{Sr} 9 b(2), \operatorname{Sr} 24$ (2) and Sr30 (17) singly. Thirty entries carried Sr8a in combinations with one to three genes including $\operatorname{Sr} 9 b, \operatorname{Sr} 12, \operatorname{Sr} 17, \operatorname{Sr} 23, \operatorname{Sr} 30$ and $\operatorname{Sr} 38$. Similarly, $\mathrm{Sr} 8 \mathrm{~b}$ was postulated in nine entries in combination with $\operatorname{Sr} 9 b$, Sr12, $\operatorname{Sr} 17$ and $\operatorname{Sr} 30$. Sr30, Sr38, Sr17 and $S r 8 a$ were among the most predominant stem rust resistances genes detected in this nursery. Sr24 was postulated in seven entries and Sr31 in four lines.

Table 4. Proportion of resistant and susceptible entries when tested against P. graminis f. sp. tritici pathotypes

\begin{tabular}{lllll}
\hline Pgt Race & Resistant & \multicolumn{3}{l}{ Susceptible } \\
\cline { 2 - 5 } & Number of entries & Percentage & Number of entries & Percentage \\
\hline $34-1,2,3,4,5,6,7(103)$ & 91 & 95.8 & 4 & 4.2 \\
$34-1,2,3,6,7,(8), 9(205)$ & 67 & 70.5 & 28 & 29.5 \\
$34-1,2,3,5,7,8,9(206)$ & 69 & 72.6 & 26 & 27.4 \\
$343-1,2,3,5,6,(8), 9(465)$ & 51 & 53.7 & 44 & 46.3 \\
$98-1,2,(3),(5), 6(279)$ & 80 & 84.2 & 15 & 15.8 \\
$34-1,2,7+\operatorname{Sr} 38(565)$ Hot & 91 & 95.8 & 4 & 4.2 \\
$34-1,2,7+\operatorname{Sr38}(565)$ Cold & 92 & 96.8 & 3 & 3.2 \\
$34-2,4,5,7,11(99)$ Hot & 91 & 95.8 & 4 & 4.2 \\
$34-2,4,5,7,11(99)$ Cold & 93 & 97.9 & 2 & 2.1 \\
All tested races & 73 & 76.8 & 22 & 23.2 \\
\hline
\end{tabular}

Table 5. Proportion of resistant and susceptible wheat entries when tested against five $P$. striformis f. sp. tritici pathotypes

\begin{tabular}{lllll}
\hline Pst Race & Resistant & \multicolumn{3}{l}{ Susceptible } \\
\cline { 2 - 5 } & Number of entries & Percentage & Number of entries & Percentage \\
\hline 134 E16A+Yr17+(599) & 54 & 56.8 & 41 & 43.2 \\
134 E16A+Yr17+Yr27 (617) & 39 & 41.1 & 56 & 58.9 \\
110 E143A+(444) & 54 & 56.8 & 41 & 43.2 \\
108 E141A+(420) & 52 & 54.7 & 43 & 45.3 \\
104 E137A+(414) & 33 & 34.7 & 62 & 65.3 \\
All tested races & 8 & 8.4 & 87 & 91.6 \\
\hline
\end{tabular}


Table 6. Seedling stem rust resistance variation in the CIMMYT international wheat screening nursery

\begin{tabular}{|c|c|c|}
\hline Resistance genes detected & Frequency & Entries \\
\hline Sr8a & 1 & (20:ZWW12) \\
\hline $\operatorname{Sr} 8 a, \operatorname{Sr} 9 b, \operatorname{Sr} 17, \operatorname{Sr} 38$ & 1 & (138:ZWW12) \\
\hline $\begin{array}{l}\operatorname{Sr} 8 a, \operatorname{Sr} 9 g, \operatorname{Sr} 12, \operatorname{Sr} 17 \\
\operatorname{Sr} 30, \operatorname{Sr} 38\end{array}$ & 1 & (11:ZWW12) \\
\hline $\begin{array}{l}\operatorname{Sr} 8 a, \operatorname{Sr} 12, \operatorname{Sr} 17, \operatorname{Sr} 30 \text {, } \\
\text { unknown }\end{array}$ & 1 & $(13: Z W W 12)$ \\
\hline $\operatorname{Sr} 8 a, \operatorname{Sr} 12, \operatorname{Sr} 30$ & 2 & (88:ZWW12, 101:ZWW12) \\
\hline $\operatorname{Sr} 8 a, \operatorname{Sr} 12, \operatorname{Sr} 17, \operatorname{Sr} 30$ & 1 & (1:ZWW12) \\
\hline $\operatorname{Sr} 8 a, \operatorname{Sr} 17, \operatorname{Sr} 30, \operatorname{Sr} 38$ & 5 & $\begin{array}{l}\text { (8:ZWW12, 10:ZWW12, 32:ZWW12, 35:ZWW12, } \\
\text { 97:ZWW12) }\end{array}$ \\
\hline $\operatorname{Sr} 8 a, \operatorname{Sr} 17, \operatorname{Sr} 30$ & 4 & (2:ZWW12, 3:ZWW12, 25:ZWW12, 131:ZWW12) \\
\hline $\operatorname{Sr} 8 a, \operatorname{Sr} 17, \operatorname{Sr} 38$ & 5 & $\begin{array}{l}\text { (52:ZWW12, 53:ZWW12, 54:ZWW12, 57:ZWW12, } \\
\text { 58:ZWW12) }\end{array}$ \\
\hline $\operatorname{Sr} 8 a, \operatorname{Sr} 23, \operatorname{Sr} 30$ & 1 & (4:ZWW12) \\
\hline $\operatorname{Sr} 8 a, \operatorname{Sr} 30, \operatorname{Sr} 38$ & 1 & $(30: Z W W 12)$ \\
\hline Sr8a, Sr30 & 7 & $\begin{array}{l}\text { (68:ZWW12, 69:ZWW12, 70:ZWW12, 98:ZWW12, } \\
\text { 107:ZWW12, 108:ZWW12, 145:ZWW12) }\end{array}$ \\
\hline $\operatorname{Sr} 8 b$ & 1 & $(116: Z W W 12)$ \\
\hline $\operatorname{Sr} 8 b, \operatorname{Sr} 9 b$ & 2 & (117:ZWW12, 119:ZWW12) \\
\hline Sr $8 b, \operatorname{Sr} 9 b, \operatorname{Sr} 12$ & 1 & $(120: Z W W 12)$ \\
\hline $\mathrm{Sr} 8 b, \mathrm{Sr} 9 b, \mathrm{Sr} 12+$ & 1 & (34:ZWW12) \\
\hline $\operatorname{Sr} 8 b, \operatorname{Sr} 9 b, \operatorname{Sr} 17$ & 1 & (51:ZWW12) \\
\hline $\operatorname{Sr} 8 b, \operatorname{Sr} 12, \operatorname{Sr} 30$ & 2 & (112:ZWW12, 113:ZWW12) \\
\hline $\mathrm{Sr} 8 b, \mathrm{Sr} 12$ & 1 & $(114: Z W W 12)$ \\
\hline $\operatorname{Sr} 8 b, \operatorname{Sr} 17$ & 1 & (21:ZWW12) \\
\hline Sr8b, Sr30, unknown & 1 & (111:ZWW12) \\
\hline $\mathrm{Sr} 9 \mathrm{~b}$ & 2 & (37:ZWW12, 122:ZWW12) \\
\hline $\operatorname{Sr} 9 b, \operatorname{Sr} 30$ & 1 & (87:ZWW12) \\
\hline $\operatorname{Sr} 12, \operatorname{Sr} 17, \operatorname{Sr} 30, \operatorname{Sr} 38$ & 1 & (26:ZWW12) \\
\hline $\operatorname{Sr} 12, \operatorname{Sr} 36, \operatorname{Sr} 38$ & 1 & (9:ZWW12) \\
\hline $\operatorname{Sr} 17, \operatorname{Sr} 24$ & 1 & (137:ZWW12) \\
\hline $\operatorname{Sr} 17, \operatorname{Sr} 30$ & 5 & $\begin{array}{l}\text { (12:ZWW12, 39:ZWW12, 46:ZWW12, 132:ZWW12, } \\
\text { 136:ZWW12) }\end{array}$ \\
\hline Sr17, Sr30, unknown & 1 & (134:ZWW12) \\
\hline $\operatorname{Sr} 17, \operatorname{Sr} 30, \operatorname{Sr} 38$ & 2 & (28:ZWW12, 31:ZWW12) \\
\hline $\operatorname{Sr} 17+$ & 1 & (18:ZWW12) \\
\hline $\operatorname{Sr} 23, \operatorname{Sr} 30$ & 1 & (43:ZWW12) \\
\hline $\mathrm{Sr} 24$ & 2 & (42:ZWW12, 44:ZWW12) \\
\hline $\operatorname{Sr} 24+$ & 1 & (129:ZWW12) \\
\hline $\operatorname{Sr} 24, \operatorname{Sr} 31, \operatorname{Sr} 38$ & 1 & (62:ZWW12) \\
\hline $\operatorname{Sr} 24, \operatorname{Sr} 38$ & 2 & (19:ZWW12, 33ZWW12) \\
\hline $\operatorname{Sr} 26, \operatorname{Sr} 38$ & 1 & (55:ZWW12) \\
\hline $\operatorname{Sr} 30$ & 17 & $\begin{array}{l}\text { (5:ZWW12, 15:ZWW12, 16:ZWW12, 17:ZWW12, } \\
\text { 23:ZWW12, 24:ZWW12, 29:ZWW12, 48:ZWW12, } \\
\text { 59:ZWW12, 61:ZWW12, 67:ZWW12, 77:ZWW12, } \\
\text { 78:ZWW12, 85:ZWW12, 109:ZWW12, 118:ZWW12, } \\
\text { 126:ZWW12) }\end{array}$ \\
\hline Sr30, unknown & 3 & (45:ZWW12, 49:ZWW12, 102:ZWW12) \\
\hline Sr30, Sr38, unknown & 1 & $(130: Z W W 12)$ \\
\hline
\end{tabular}




\begin{tabular}{lll}
\hline Resistance genes detected & Frequency & Entries \\
\hline Sr31, Sr38 & 3 & $(56: Z W W 12,63: Z W W 12,64:$ ZWW12) \\
Sr38, unknown & 7 & $(36: Z W W 12,100:$ ZWW12, 127:ZWW12, \\
& & $128:$ ZWW12, 140:ZWW12, 141:ZWW12, \\
& & $14:$ ZWW12) \\
\hline
\end{tabular}

Table 7. Seedling stripe rust resistance variation in the CIMMYT international wheat screening nursery

\begin{tabular}{|c|c|c|}
\hline $\begin{array}{l}\text { Resistance genes } \\
\text { postulated }\end{array}$ & Frequency & Entry \\
\hline Yr3 & 11 & $\begin{array}{l}\text { (1:ZWW12, 2:ZWW12, 17:ZWW12, 20:ZWW12, } \\
\text { 21:ZWW12, 42:ZWW12, 44:ZWW12, 45:ZWW12, } \\
\text { 113:ZWW12, 119:ZWW12, 131:ZWW12) }\end{array}$ \\
\hline Yr3, unknown & 2 & $(35: Z W W 12,111: Z W W 12)$ \\
\hline $\operatorname{Yr} 3, \operatorname{Yr} 6$ & 1 & (85:ZWW12) \\
\hline $\operatorname{Yr} 3, \operatorname{Yr} 9, \operatorname{Yr} 17$ & 1 & $(64: Z W W 12)$ \\
\hline $\operatorname{Yr} 4, \operatorname{Yr} 9, \operatorname{Yr} 17$ & 1 & $(63: Z W W 12)$ \\
\hline Yr6 & 6 & $\begin{array}{l}\text { (29:ZWW12, 37:ZWW12, 52:ZWW12, 98:ZWW12, } \\
\text { 101:ZWW12, 109:ZWW12, ) }\end{array}$ \\
\hline $\operatorname{Yr} 9$, unknown & 1 & $(18: Z W W 12)$ \\
\hline $\operatorname{Yr} 9, \operatorname{Yr} 17$ & 1 & $(62: Z W W 12)$ \\
\hline $\operatorname{Yr} 17, \operatorname{Yr} 27$ & 24 & $\begin{array}{l}\text { (9:ZWW12, 10:ZWW12, 11:ZWW12, 26:ZWW12, } \\
\text { 28:ZWW12, 30:ZWW12, 31:ZWW12, 32:ZWW12, } \\
\text { 33:ZWW12, 36:ZWW12, 54:ZWW12, 55:ZWW12, } \\
\text { 56:ZWW12, 57:ZWW12, 58:ZWW12, 97:ZWW12, } \\
\text { 100:ZWW12, 127:ZWW12, 128:ZWW12, 130:ZWW12, } \\
\text { 138:ZWW12, 140:ZWW12, 141:ZWW12, 142:ZWW12) } \\
\text { (8:ZWW12, 19:ZWW12) }\end{array}$ \\
\hline Yr27 & 8 & $\begin{array}{l}\text { (4:ZWW12, 5:ZWW12, 16:ZWW12,39:ZWW12, } \\
\text { 88:ZWW12, 108:ZWW12, 136:ZWW12, 145:ZWW12) }\end{array}$ \\
\hline Yr27, unknown & 1 & $(87: Z W W 12)$ \\
\hline Yr34 & 3 & (114:ZWW12, 116:ZWW12, 117:ZWW12) \\
\hline Yr34, unknown & 1 & $(112: Z W W 12)$ \\
\hline Unknown & 7 & $\begin{array}{l}\text { (3:ZWW12, 13:ZWW12, 18:ZWW12, 34:ZWW12, } \\
\text { 61:ZWW12, 107:ZWW12, 118:ZWW12) }\end{array}$ \\
\hline None & 26 & $\begin{array}{l}\text { (12:ZWW12, 15:ZWW12, 23:ZWW12, 24:ZWW12, } \\
\text { 25:ZWW12, 43:ZWW12, 46:ZWW12, 48:ZWW12, } \\
\text { 49:ZWW12, 51:ZWW12, 53:ZWW12, 59:ZWW12, } \\
\text { 67:ZWW12, 68:ZWW12, 69:ZWW12, 70:ZWW12, } \\
\text { 77:ZWW12, 78:ZWW12, 102:ZWW12, 120:ZWW12, } \\
\text { 122:ZWW12, 126:ZWW12, 129:ZWW12, 132:ZWW12, } \\
\text { 134:ZWW12, 137:ZWW12) }\end{array}$ \\
\hline
\end{tabular}

\section{Stripe rust}

Eight $(8.4 \%)$ of the 95 tested wheat entries showed different resistance levels to the five races of stripe rust at the seedling stage (Table 5). The rest of $87(91.6 \%)$ wheat entries showed varying 
levels of susceptibility. Stripe rust multi-pathotype testing results are summarized in Table 1 and 7. Screening with five different Pst pathotypes detected seven seedling stripe rust resistance genes (Yr3, Yr4, Yr6, Yr9, Yr17, Yr27 and Yr34) either singly or in combinations. Yr3 was present singly in 11 entries and in combination with $Y r 6$ in one line (85:ZWW12). $Y r 3$ in combination with an additional gene was postulated in entries 35:ZWW12 and 111:ZWW12. $Y r 3$ in combination with $Y r 9$ and $Y r 17$ was detected in entry 64:ZWW12. Stripe rust resistance gene $Y r 4$ in combination with $Y r 9$ and $Y r 17$ was postulated in entry 63:ZWW12. Yr6 was detected singly in six entries (Table 1, 7). $Y r 9$ and $Y r 17$ were postulated in one line (62:ZWW12). Additionally, Yr17 was present singly in 24 entries and in combinations with $Y r 27$ in two lines. Likewise, $Y$ r27 was detected singly in eight entries and in combination with additional unknown resistance in one line (87:ZWW12). On the other hand, Yr34 was postulated singly in three lines and in combination with extra unknown resistance in one entry. Seven entries carried resistance genes that could not be detected by the array of Pst pathotypes used. No seedling stripe rust resistance genes were postulated in 26 entries. ASR genes $\operatorname{Yr} 17(32 \%)$ followed by $Y r 3(16 \%)$ and $Y r 27(12 \%)$ were the most frequent resistance genes detected. $Y r 4(1 \%), Y r 9(3 \%)$ and $Y r 34$ $(4 \%)$ were the least frequently detected.

\section{Molecular marker genotyping}

The marker cs $S r 2$, detected APR gene $S r 2$ in four entries (Hope allele; 172bp, 112bp and 53bp), whereas 80 entries amplified the Marquis type allele (225bp and 112bp) after digestion with the restriction enzyme BspHI. Eleven entries did not amply any product. Out of the 95 entries genotyped with the dominant STS marker Sr24\#12, only nine entries and the positive control Janz produced a $500 \mathrm{bp}$ amplicon associated with $\operatorname{Sr} 24$, whereas no amplification was observed in the remaining 86 entries. The marker iag95 detected Sr31/Yr9/Lr26 in three entries, while the marker Ventriup + LN2 confirmed the presence of Sr38/Yr17/Lr37 in 32 entries. One genotype was confirmed to carry $Y r 4$ when genotyped with SSR marker barc75. Based on marker csLV 34, 22 entries were observed to carry the pleiotropic APR gene Yr18/Lr34/Sr57 (Table 1). Of the 22 entries confirmed to carry $\operatorname{Yr} 18$, three entries (24:ZWW12, 53:ZWW12 and 132:ZWW12) were susceptible at the seedling stage.

\section{Discussion}

Successful deployment of rust resistance genes depends on a better understanding of the genetic diversity among donor sources (Bariana et al., 2007a). The main objective of this study was to assess the genetic diversity for stem rust and stripe rust resistance in an international wheat screening nursery. Strategic deployment of stem rust resistance started in Australia with the release of cultivars Hofed and Fedweb in 1937 (Macindoe and Walkden-Brown, 1968). The Sr11 carrying cultivar Gabo was released in 1945 and became backbone of the CIMMYT's wheat improvement program due to it photoperiod insensitivity.

Multi-pathotype evaluations identified stem rust ASR genes $\mathrm{Sr} 8$ a, Sr8b, SrOb, Sr12, Sr17, Sr23, Sr24, Sr30, Sr31 and Sr38 and stripe rust resistance genes Yr3, Yr4, Yr6, Yr9, Yr17, Yr27 and Yr34 either singly or in combinations. Unfortunately, most of these genes are not effective individually against at least one of the Pgt and Pst pathotypes worldwide (Singh et al., 2008b). Postulation of the above-mentioned stem rust and stripe rust seedling resistance genes is expected because these seem to be fixed in breeding populations due to their widespread use. For example; Singh et al., (2008a) postulated eight stem rust resistance genes (Sr5, Sr8a, SrOg, 
$\operatorname{Sr12,} \operatorname{Sr} 30, \operatorname{Sr} 31, \operatorname{Sr} 36$ and $\operatorname{Sr} 38)$ and seven stripe rust resistance genes (Yr1, $\operatorname{Yr} 6, \operatorname{Yr} 7, \mathrm{Yr}$, $\operatorname{Yr17}$, Yr27, YrHVII) either singly or in combinations in wheat cultivars from the United Kingdom. Admassu et al., (2012), reported 11 stem rust resistance genes $(\operatorname{Sr} 5, \operatorname{Sr} 7 a$, $\operatorname{Sr} 7 b$, $\operatorname{Sr} 8 a$, $\operatorname{Sr} 9 e$, $\operatorname{Sr} 11$, $\operatorname{Sr} 21, \operatorname{Sr} 27, \operatorname{Sr} 29$, Sr30 and Sr37) either singly or in combinations in durum and bread wheat cultivars and breeding lines from Ethiopia. Spanic et al., (2015) reported four stem rust resistance genes (Sr8a, Sr31, Sr36, Sr38) in Croatian wheat cultivars. Kolmer et al., (2007) when comparing the frequency of stem rust resistance genes in United States winter and spring wheats found that resistance genes $\operatorname{Sr} 2, \operatorname{Sr} 6, \operatorname{Sr} 17, \operatorname{Sr} 24, \operatorname{Sr} 31, \operatorname{Sr} 36$ and $\operatorname{SrTmp}$ were common in winter wheats, while genes $\operatorname{Sr} 6, \operatorname{Sr} 9 b, \operatorname{Sr} 11$ and $\operatorname{Sr} 17$ were more frequent in spring wheats. This study found $\operatorname{Sr} 8$ a, Sr12, Sr17, $\operatorname{Sr} 30$ and $\operatorname{Sr} 38$ to be more common in CIMMYT spring wheat nursery. Postulation of common genes in different studies is attributed to the use of CIMMYT germplasm directly or as parents in many countries (Ortiz et al., 2007; 2008; Pretorius et al., 2015). It is estimated that about $70-80 \%$ of spring wheat cultivars released in the developing world are CIMMYT lines or lines derived from CIMMYT parents (Wang et al., 2003; Ortiz et al., 2007) indicating the level of international effort to breed against deadly pathogens and wider adaptation, and the importance of CGIAR centres like CIMMYT in providing improved germplasm to national breeding programs in developing countries.

Sr30 was the most frequent stem rust seedling resistance gene. Although a Pgt pathotype virulent on Sr30 was reported in Eastern Australia by Park and Wellings, (1992), it is still effective against commercially important pathotypes (Bariana et al., 2007b). Sr30 virulence was also reported in many other countries including Spain, Ethiopia, Turkey, Pakistan and South American countries (Huerta-Espino, 1992). Sr30 is the backbone of the CIMMYT and Australian germplasm (McIntosh et al., 1995, Bariana et al., 2007). Other stem rust resistance genes detected in high frequency were $\operatorname{Sr} 38(34 \%), \operatorname{Sr} 8 a(33 \%), \operatorname{Sr} 17(33 \%)$ and $\operatorname{Sr} 12(13 \%)$. Virulence for Sr38 was first detected in Western Australia in 2001 (Park, 2008); however, this gene is still being used in breeding programs because of its linkage with cereal cyst nematode gene Cre5 (Jahier et al., 2001). Resistance genes Sr31, Sr24 and Sr23 were detected at a very low frequency in this nursery. The virulence in $\mathrm{Ug} 99$ and its variants on these genes has possibly been responsible for this trend (Singh et al., 2015).

It is surprising not to detect stem rust resistance genes $\operatorname{Sr} 13, \operatorname{Sr} 15$ and $\operatorname{Sr} 36$ in this study. $\operatorname{Sr} 13$ has not been deployed widely, except in some Australian cultivars such as, Miskle and Machete (H.S. Bariana unpublished results). The incorporation of Sr15 and Sr36 in CIMMYT's wheat improvement program is likely to happen due to association of genomic regions carrying these genes with root lesion nematode resistance gene Rlnn1 (Jayatilake et al., 2013) and crown rot resistance respectively. Stem rust resistance genes $\operatorname{Sr} 33$ and $S r 45$ were deployed in Australian wheat cultivars Lorikeet and Thornbill, respectively (H.S. Bariana unpublished results). Overall, the cultivars identified with known stem rust resistance genes will provide valuable genetic material for breeding resistant wheat cultivars.

The most predominant seedling stripe rust resistance genes detected were $\operatorname{Yr} 17$ (34\%), $\mathrm{Yr} 3$ (16\%) and Yr27 (12\%). Pathan, (2003) reported the presence of Yr17/Lr37/Sr38 (VPM) cluster in many European wheats. The VPM segment has been widely deployed in commercial cultivars in many parts of the world including Australia (Park, 2008). The popularity of this useful translocation has declined due to reported virulences for all the three rust resistance genes (Singh et al., 2008a). Virulence for Yr17 was first detected in eastern Australia in 1999 and was thought to have originated from an existing pathotype via mutation (Wellings, 2007) and by 2006 a pathotype with combined virulence for Yr17, Yr6, Yr7 and YrA was identified (Wellings, 2007). 
The second highly frequent stripe rust resistance gene in this study was $\mathrm{Yr}^{3}(16 \%)$. Yr3 was also postulated in CIMMYT wheat germplasm by Dubin et al., (1989). Yr3 was not an important gene for Australia until the detection of WA pathotype in 2002, which carried virulence for Yr6, Yr7, Yr8, Yr9, and YrA and avirulence for Yr3 and Yr4 (Wellings et al., 2003; Wellings and Kandel, 2004). The effectiveness of the 1BL.1RS (Lr26/Yr9/Sr31) translocation in protecting wheat against stem rust for over 30 years before the detection of Ug99 in 1998 (Pretorius et al., 2000), led to high frequency of these three genes in most wheats globally (Singh et al., 2008b). High proportions of $\mathrm{Yr} 9$ have been reported in Chinese wheat cultivars (Zeng et al., 2014). Pathan et al., (2008) also reported a high frequency of $Y r 9$ in European wheats. Singh et al., (2014) postulated the stripe rust resistance gene $Y r 9$ (1BL.1RS rye-derived) in 58\% of the $12^{\text {th }}$ High Temperature Wheat Yield Trial (12 ${ }^{\text {th }}$ HTWYT), 17\% of the $22^{\text {nd }}$ Semi-Arid Wheat Screening Nursery (22nd SAWSN) and 2\% entries of the $1^{\text {st }}$ Australian Special Nursery (1st ASN). On the contrary, the $Y r 9$-rye translocation was detected in only $3 \%$ of the entries in the nursery screened in the present study. The declining frequency of $Y r 9$ in CIMMYT germplasm could be due to the reported virulence for $S r 31$ and $Y r 9$ located on the IBL-1RS translocation (Pretorius et al., 2000; Wellings et al., 2003). Seedling stripe rust resistance gene Yr27 was present in a number of CIMMYT wheats including Ciano 79, Nacozari 76, Crow, Tesia 79, Opata 85, Bacanora 88, Bakhtawar, WH542, Atrak, Memof, PBW343, MH97, Chamaran, Kubsa, and Shirudi (Wellings, 1992). However, the outbreak of Yr27 virulent pathotype in 2010-2013 caused significant yield losses in Afghanistan, Azerbaijan, Ethiopia, Iran, Iraq, Kenya, Morocco, Syria, Turkey and Uzbekistan (Singh et al., 2012; FAO, 2014). The ineffectiveness of this widely deployed resistance gene posed a serious threat to food security and livelihoods of resourcepoor farmers and their communities.

Yr34 was mapped on chromosome 5AL of wheat genotype WAWHT2046 (Bariana et al., 2006). It is effective against $134 \mathrm{E} 16 \mathrm{~A}+$ and its variants. It was only present in $4 \%$ of the entries. Seven entries carried resistance that could not be postulated by the array of Pst pathotypes used in this study indicating that they carry either new (uncharacterized) genes effective against all pathotypes used in this study or combinations of genes. Twenty seven percent of entries were susceptible at the seedling stage to all Pst pathotypes used and field testing is recommended to determine their field response to stripe rust at the adult plant stage.

This investigation explained the diversity in CIMMYT wheat germplasm through postulation of known genes for resistance to stem rust and stripe rust diseases using phenotypic assessments against several pathotypes of each rust pathogen and markers linked with APR genes. Genotypes carrying potentially new uncharacterized ASR genes for stem rust and stripe rust resistance against Australian Pst pathotypes were identified for formal genetic analysis. The genotypes with good level of resistance to the two rusts have been identified for incorporation into breeding programs as donor parents. The information presented in this study is useful for wheat breeders to devise strategies for achieving durable rust control.

\section{Acknowledgements}

The authors are grateful to NARO-Uganda and the Grains Research and Development Corporation (GRDC) Australia for funding this study. 


\section{References}

Admassu, B., Friedt, W. and Ordon, F. 2012. Stem rust seedling resistance genes in Ethiopian wheat cultivars and breeding lines. African Crop Science Journal, 20(3):149-162.

Ali, S., Rodriguez-Algaba, J., Thach, T., Sørensen, C.K., Hansen, J.G., Lassen, P., Nazari, K., Hodson, D.P., Justesen, A.F. and Hovmøller, M.S. 2017. Yellow rust epidemics worldwide were caused by pathogen races from divergent genetic lineages. Frontiers in Plant Science, 8, p.1057.

Ali, S., Gladieux, P., Leconte, M., Gautier, A., Justesen, A.F., Hovmøller, M.S., Enjalbert, J. and de Vallavieille-Pope, C. 2014. Origin, migration routes and worldwide population genetic structure of the wheat yellow rust pathogen Puccinia strïformis f. sp. tritici. PLoS pathogens, 10(1), p.e1003903.

Bansal, U., Bariana, H., Wong, D., Randhawa, M., Wicker, T., Hayden, M. and Keller, B. 2014. Molecular mapping of an adult plant stem rust resistance gene Sr56 in winter wheat cultivar Arina. Theoretical and applied genetics, 127(6):1441-1448.

Bansal, U.K., Muhammad, S., Forrest, K.L., Hayden, M.J. and Bariana, H.S. 2015. Mapping of a new stem rust resistance gene $S \mathrm{r} 49$ in chromosome $5 \mathrm{~B}$ of wheat. Theoretical and applied genetics, 128(10):2113-2119.

Bariana, H.S. 2003. Breeding for disease resistance. In: Thomas B (ed) Encyclopaedia of Applied Plant Sciences, Academic Press, Harcourt, UK, pp. 244-253

Bariana, H.S., Brown, G.N., Bansal, U.K., Miah, H., Standen, G.E. and Lu, M. 2007a. Breeding triple rust resistant wheat cultivars for Australia using conventional and marker-assisted selection technologies. Australian Journal of Agricultural Research, 58(6):576-587.

Bariana, H.S. and McIntosh, R.A. 1995. Genetics of adult plant stripe rust resistance in four Australian wheats and the French cultivar 'Hybride-de-Bersee'. Plant Breeding, 114(6):485491.

Bariana, H.S., Miah, H., Brown, G.N., Willey, N. and Lehmensiek, A. 2007b. Molecular mapping of durable rust resistance in wheat and its implication in breeding. In Wheat production in stressed environments (pp. 723-728). Springer, Dordrecht.

Bariana, H.S., Parry, N., Barclay, I.R., Loughman, R., McLean, R.J., Shankar, M., Wilson, R.E., Willey, N.J. and Francki, M., 2006. Identification and characterization of stripe rust resistance gene Yr34 in common wheat. Theoretical and Applied Genetics, 112(6):1143-1148.

Bernardo, A.N., Bowden, R.L., Rouse, M.N., Newcomb, M.S., Marshall, D.S. and Bai, G. 2013. Validation of molecular markers for new stem rust resistance genes in US hard winter wheat. Crop Science, 53(3):755-764.

Bhavani, S., Hodson, D.P., Huerta-Espino, J., Randhawa, M.S. and Singh, R.P. 2019. Progress in breeding for resistance to $\mathrm{Ug} 99$ and other races of the stem rust fungus in CIMMYT wheat germplasm. Front Agric Sci Eng. 2019; 6: 210-224.

Carmona, M., Sautua, F., Pérez-Hérnandez, O. and Reis, E.M. 2020. Role of fungicide applications on the integrated management of wheat stripe rust. Frontiers in Plant Science, 11:733.

Chen, X. 2020. Pathogens which threaten food security: Puccinia striiformis, the wheat stripe rust pathogen. Food Security, 12(2):239-251.

Chen, W., Wellings, C., Chen, X., Kang, Z. and Liu, T. 2014. Wheat stripe (yellow) rust caused by P uccinia striiformis f. sp. tritici. Molecular plant pathology, 15(5):433-446.

Chen, X.M. 2005. Epidemiology and control of stripe rust (Puccinia striiformis f. sp. Tritici) on wheat. Canadian journal of plant pathology, 27 (3):314-337. 
Dubin, H.J., Johnson, R. and Stubbs, R.W. 1989. Postulated genes for resistance to stripe rust in selected CIMMYT and related wheats. Plant disease, 73(6):472-475.

Ellis, J.G., Lagudah, E.S., Spielmeyer, W. and Dodds, P.N. 2014. The past, present and future of breeding rust resistant wheat. Frontiers in plant science, 5:641.

FAO 2014. Wheat rust diseases global program 2014-2017: Food chain crisis, management framework, plant protection. Rome, 1-8.

Helguera, M., Khan, I.A., Kolmer, J., Lijavetzky, D., Zhong-Qi, L. and Dubcovsky, J., 2003. PCR assays for the Lr37-Yr17-Sr38 cluster of rust resistance genes and their use to develop isogenic hard red spring wheat lines. Crop Science, 43(5):1839-1847.

Hovmøller, M.S. and Justesen, A.F. 2007. Appearance of atypical Puccinia striiformis f. sp. tritici phenotypes in north-western Europe. Australian Journal of Agricultural Research, 58(6):518-524.

Huerta-Espino, J. 1992. Analysis of wheat leaf and stem rust virulence on a worldwide basis (Doctoral dissertation, University of Minnesota).

Jahier, J., Abelard, P., Tanguy, M., Dedryver, F., Rivoal, R., Khatkar, S., Bariana, H.S. and Koebner, R. 2001. The Aegilops ventricosa segment on chromosome 2AS of the wheat cultivar 'VPM1'carries the cereal cyst nematode resistance gene Cre5. Plant Breeding, 120(2):125-128.

Jayatilake, D.V., Tucker, E.J., Bariana, H., Kuchel, H., Edwards, J., McKay, A.C., Chalmers, K. and Mather, D.E. 2013. Genetic mapping and marker development for resistance of wheat against the root lesion nematode Pratylenchus neglectus. BMC plant biology, 13(1):1-12.

Kang, Z., Zhao, J., Han, D., Zhang, H., Wang, X., Wang, C., Han, Q., Guo, J. and Huang, L. 2010, May. Status of wheat rust research and control in China. In BGRI 2010 technical workshop oral presentations (p. 50).

Kolmer, J.A. 2003. Postulation of leaf rust resistance genes in selected soft red winter wheats. Crop science, 43(4):1266-1274.

Kolmer, J.A., Jin, Y. and Long, D.L. 2007. Wheat leaf and stem rust in the United States. Australian Journal of Agricultural Research, 58(6):631-638.

Kolmer, J.A., Hanzalova, A., Goyeau, H., Bayles, R. and Morgounov, A. 2013. Genetic differentiation of the wheat leaf rust fungus Puccinia triticina in Europe. Plant Pathology, 62(1):21-31.

Kou, Y. and Wang, S. 2010. Broad-spectrum and durability: understanding of quantitative disease resistance. Current opinion in plant biology, 13(2):181-185.

Lagudah, E.S., McFadden, H., Singh, R.P., Huerta-Espino, J., Bariana, H.S. and Spielmeyer, W. 2006. Molecular genetic characterization of the Lr34/Yr18 slow rusting resistance gene region in wheat. Theoretical and Applied Genetics, 114(1):21-30.

Macharia, J.K., Wanyera, R. and Kilonzo, S. 2013. Evaluation of fungicides for controlling stem rust race Ug99 on bread wheat. Journal of Agricultural Science and Technology. A, 3(5A):404.

Macindoe, S.L. and Brown, C.W. 1968. Wheat Breeding and Varieties in Australia. Sydney. New South Wales: Division of Plant Industry, NSW Department of Agriculture, Science Bulletin No. 76.

Mago, R., Bariana, H.S., Dundas, I.S., Spielmeyer, W., Lawrence, G.J., Pryor, A.J. and Ellis, J.G. 2005. Development of PCR markers for the selection of wheat stem rust resistance genes Sr24 and Sr26 in diverse wheat germplasm. Theoretical and Applied Genetics, 111(3):496504.

Mago, R., Brown-Guedira, G., Dreisigacker, S., Breen, J., Jin, Y., Singh, R., Appels, R., Lagudah, E.S., Ellis, J. and Spielmeyer, W. 2011. An accurate DNA marker assay for stem rust resistance gene $S r 2$ in wheat. Theoretical and applied genetics, 122(4):735-744. 
Mago, R., Spielmeyer, W., Lawrence, G., Lagudah, E., Ellis, J. and Pryor, A. 2002. Identification and mapping of molecular markers linked to rust resistance genes located on chromosome 1RS of rye using wheat-rye translocation lines. Theoretical and Applied Genetics, 104(8):1317-1324.

Markell, S.G. and Milus, E.A. 2008. Emergence of a novel population of Puccinia striiformis f. sp. tritici in eastern United States. Phytopathology, 98(6):632-639.

McIntosh, R.A., Wellings, C.R. and Park, R.F. 1995. Wheat rusts: an atlas of resistance genes. CSIRO publishing.

Milus, E.A., Moon, D.E., Lee, K.D. and Mason, R.E. 2015. Race-specific adult-plant resistance in winter wheat to stripe rust and characterization of pathogen virulence patterns. Phytopathology, 105(8):1114-1122.

Murray, G.M. and Brennan, J.P. 2009. Estimating disease losses to the Australian wheat industry. Australasian Plant Pathology, 38(6):558-570.

Nazari, K., Mafi, M., Yahyaoui, A., Singh, R.P. and Park, R.F. 2009. Detection of wheat stem rust (Puccinia graminis f. sp. tritici) race TTKSK (Ug99) in Iran. Plant Disease, 93(3):317-317.

Oliver, R.P. 2014. A reassessment of the risk of rust fungi developing resistance to fungicides. Pest management science, 70(11):1641-1645.

Ortiz, R., Braun, H.J., Crossa, J., Crouch, J.H., Davenport, G., Dixon, J., Dreisigacker, S., Duveiller, E., He, Z., Huerta, J. and Joshi, A.K. 2008. Wheat genetic resources enhancement by the International Maize and Wheat Improvement Centre (CIMMYT). Genetic Resources and Crop Evolution, 55(7):1095-1140.

Ortiz, R., Trethowan, R., Ferrara, G.O., Iwanaga, M., Dodds, J.H., Crouch, J.H., Crossa, J. and Braun, H.J., 2007. High yield potential, shuttle breeding, genetic diversity, and a new international wheat improvement strategy. Euphytica, 157(3):365-384.

Park, R.F. 2008. Breeding cereals for rust resistance in Australia. Plant Pathology, 57(4):591602.

Pathan, A.K. and Park, R.F. 2007. Evaluation of seedling and adult plant resistance to stem rust in European wheat cultivars. Euphytica, 155(1):87-105.

Park, R.F. and Wellings, C.R. 1992. Pathogenic specialisation of wheat rusts in Australia and New Zealand in 1988 and 1989. Australasian Plant Pathology, 21(2):61-69.

Pretorius, Z.A., Singh, R.P., Wagoire, W.W. and Payne, T.S. 2000. Detection of virulence to wheat stem rust resistance gene Sr31 in Puccinia graminis. f. sp. tritici in Uganda. Plant Disease, 84(2):203-203.

Pretorius, Z.A., Visser, B., Terefe, T., Herselman, L., Prins, R., Soko, T., Siwale, J., Mutari, B., Selinga, T.I. and Hodson, D.P. 2015. Races of Puccinia triticina detected on wheat in Zimbabwe, Zambia and Malawi and regional germplasm responses. Australasian Plant Pathology, 44(2):217-224.

Qamar, M., Ahmad, S.D., Shah, A.H., Wellings, C.R. and Batool, F. 2008. Postulation of stripe rust resistant genes in some Australian bread wheat cultivars and their response to temperature. Pak. J. Bot, 40(6):2573-2585.

Roelfs, A.P. 1992. Rust diseases of wheat: concepts and methods of disease management. Cimmyt.

Singh, B., Bansal, U.K., Muhammad, H.M., Gill, B. and Bariana, H.S. 2014. Postulation of resistance genes and assessment of adult plant response variation for stripe rust in three international wheat nurseries. Indian J. Genet, 74(1):1-9.

Singh, D., Park, R.F., McIntosh, R.A. and Bariana, H.S. 2008a. Characterisation of stem rust and stripe rust seedling resistance genes in selected wheat cultivars from the United Kingdom. Journal of Plant Pathology, pp.553-562. 
Singh, R.P., Hodson, D.P., Huerta-Espino, J., Jin, Y., Njau, P., Wanyera, R., Herrera-Foessel, S.A. and Ward, R.W. 2008b. Will stem rust destroy the world's wheat crop? Advances in agronomy, 98:271-309.

Singh, R.P., Hodson, D.P., Huerta-Espino, J., Jin, Y., Bhavani, S., Njau, P., Herrera-Foessel, S., Singh, P.K., Singh, S. and Govindan, V. 2011a. The emergence of Ug99 races of the stem rust fungus is a threat to world wheat production. Annual review of phytopathology, 49:465-481.

Singh, R.P., Huerta-Espino, J., Bhavani, S., Herrera-Foessel, S.A., Singh, D., Singh, P.K., Velu, G., Mason, R.E., Jin, Y., Njau, P. and Crossa, J. 2011b. Race non-specific resistance to rust diseases in CIMMY'T spring wheats. Euphytica, 179(1):175-186.

Singh, R.P., Herrera-Foessel, S., Huerta-Espino, J., Singh, S., Bhavani, S., Lan, C. and Basnet, B.R. 2014. Progress towards genetics and breeding for minor genes-based resistance to Ug99 and other rusts in CIMMYT high-yielding spring wheat. Journal of Integrative Agriculture, 13(2):255-261.

Singh, R.P. and Rajaram, S. 2002. Breeding for disease resistance in wheat. FAO Plant Production and Protection Series, PP. 567

Sørensen, C.K., Hovmøller, M.S., Leconte, M., Dedryver, F. and de Vallavieille-Pope, C. 2014. New races of Puccinia striiformis found in Europe reveal race specificity of long-term effective adult plant resistance in wheat. Phytopathology, 104(10):1042-1051.

Spanic, V., Rouse, M.N., Kolmer, J.A. and Anderson, J.A. 2015. Leaf and stem seedling rust resistance in wheat cultivars grown in Croatia. Euphytica, 203(2):437-448.

Tadesse, K., Ayalew, A. and Badebo, A. 2010. Effect of fungicide on the development of wheat stem rust and yield of wheat varieties in highlands of Ethiopia. African Crop Science Journal, 18(1).

Tsilo, T.J., Chao, S., Jin, Y. and Anderson, J.A. 2009. Identification and validation of SSR markers linked to the stem rust resistance gene $\mathrm{Sr} 6$ on the short arm of chromosome 2D in wheat. Theoretical and applied genetics, 118(3):515-524.

Tsilo, T.J., Jin, Y. and Anderson, J.A. 2008. Diagnostic microsatellite markers for the detection of stem rust resistance gene $\mathrm{Sr} 36$ in diverse genetic backgrounds of wheat. Crop Science 48:253-261

Vanzetti, L.S., Campos, P., Demichelis, M., Lombardo, L.A., Aurelia, P.R., Vaschetto, L.M., Bainotti, C.T. and Helguera, M. 2011. Identification of leaf rust resistance genes in selected Argentinean bread wheat cultivars by gene postulation and molecular markers. Electronic Journal of Biotechnology, 14(3):9-9.

Vikram, P., Sehgal, D., Sharma, A., Bhavani, S., Gupta, P., Randhawa, M., Pardo, N., Basandra, D., Srivastava, P., Singh, S. and Sood, T. 2021. Genome-wide association analysis of Mexican bread wheat landraces for resistance to yellow and stem rust. PloS one, 16(1), p.e0246015.

Walter, S., Ali, S., Kemen, E., Nazari, K., Bahri, B.A., Enjalbert, J., Hansen, J.G., Brown, J.K., Sicheritz-Pontén, T., Jones, J. and de Vallavieille-Pope, C. 2016. Molecular markers for tracking the origin and worldwide distribution of invasive strains of Puccinia striiformis. Ecology and Evolution, 6(9):2790-2804.

Wang, J., Van Ginkel, M., Podlich, D., Ye, G., Trethowan, R., Pfeiffer, W., DeLacy, I.H., Cooper, M. and Rajaram, S. 2003. Comparison of two breeding strategies by computer simulation. Crop Science, 43(5):1764-1773.

Wanyera, R., Macharia, J.K., Kilonzo, S.M. and Kamundia, J.W. 2009. Foliar fungicides to control wheat stem rust, race TTKS (Ug99), in Kenya. Plant disease, 93:929-932.

Wellings, C.R. 2011. Global status of stripe rust: a review of historical and current threats. Euphytica, 179:129-141. 
Wellings, C.R., 2007. Puccinia striiformis in Australia: a review of the incursion, evolution, and adaptation of stripe rust in the period 1979-2006. Australian Journal of Agricultural Research, 58(6):567-575.

Wellings, C.R. and Kandel, K.R. 2004, August. Pathogen dynamics associated with historic stripe (yellow) rust epidemics in Australia in 2002 and 2003. In Proceedings of the 11th international cereal rusts and powdery mildews conference (pp. 22-27).

Wellings, C.R., Wright, D.G., Keiper, F. and Loughman, R. 2003. First detection of wheat stripe rust in Western Australia: evidence for a foreign incursion. Australasian Plant Pathology, 32(2):321-322.

Zeng, Q.D., Han, D.J., Wang, Q.L., Yuan, F.P., Wu, J.H., Zhang, L., Wang, X.J., Huang, L.L., Chen, X.M. and Kang, Z.S. 2014. Stripe rust resistance and genes in Chinese wheat cultivars and breeding lines. Euphytica, 196(2):271-284. 